\title{
Smart Manufacturing Process of Carbon-Based Low-Dimensional Structures and Fiber-Reinforced Polymer Composites for Engineering Applications
}

\author{
V.K. Srivastava, Pramod Kumar Jain, Parshant Kumar, Alessandro Pegoretti, and Chris R. Bowen \\ (Submitted June 16, 2019; in revised form December 28, 2019; published online July 22, 2020)
}

\begin{abstract}
Reinforced polymers have gained a significant interest in the research community in recent years due to their enhanced performance as compared to their parent polymers. Nanosized reinforcement-based polymer composites have shown a considerable enhancement in performance as compared to micro-/macrosized fillers. But achieving a high degree of filler alignment in nanocomposites via conventional manufacturing techniques continues to be a challenging task. Additive manufacturing (AM) is a possible solution to this challenge and has undergone a significant development in recent years. AM is an efficient technique to fabricate nanocomposites in complex shapes which uses a layer-by-layer method to fabricate materials and thereby eliminates the need for multi-step processing and the use of fasteners/joints to fabricate complex structures. Due to a wide range of properties and the existence of carbon in a variety of forms, carbon fillerbased polymer matrix composites are suitable for applications in several technical sectors. AM of carbonbased structures and fiber-reinforced polymer composites enables the production of highly customized parts with a high degree of filler alignment. AM techniques which are generally employed for the fabrication of carbon-based fillers/fiber-reinforced polymer composites are fused deposition modeling, stereolithography, direct ink writing and selective laser sintering. Although AM techniques are efficient, some critical issues such as voids and defects between interlayers, the low adhesion strength between filaments, part curling and insufficient curing of layers need to be addressed to utilize its full potential.
\end{abstract}

Keywords additive manufacturing, carbon, composites, polymers

\section{Introduction}

The physical and mechanical properties of composite material can be tailored by an appropriate selection of its constituents. Designing a composite at the microstructure level and varying the processing techniques may also be used to tailor its properties (Ref 1). To design a composite at a microstructural level, a variety of reinforcements can be considered; these include discrete particles (Ref 2), unidirectional short (Ref 3 ) and continuous fibers (Ref 4), twodimensional woven fabric (Ref 5), three-dimensional fiber arrangement with stitched layers (Ref 6), and randomly oriented fibers (Ref 7). The filler orientation (Ref 8) is also taken into consideration. However, using conventional techniques (i.e., hand lay-up and resin transfer molding) to manufacture a composite structure with a designed architecture can impose some technological barriers such as curvature of laminates (Ref 1). Thus, its performance may exhibit some

V.K. Srivastava and Parshant Kumar, Department of Mechanical Engineering, Indian Institute of Technology (Banaras Hindu University), Varanasi 221005, India; Pramod Kumar Jain, Indian Institute of Technology (Banaras Hindu University), Varanasi 221005, India; Alessandro Pegoretti, Department of Industrial Engineering, University of Trento, Trento, Italy; and Chris R. Bowen, Department of Mechanical Engineering, University of Bath, Bath, UK. Contact e-mail: vijayks210@gmail.com.

\begin{tabular}{|ll|}
\hline & \multicolumn{1}{c|}{ Abbreviations } \\
\hline $\mathrm{AM}$ & Additive manufacturing \\
FDM & Fused deposition modeling \\
$\mathrm{SLA}$ & Stereolithography \\
$\mathrm{DIW}$ & Direct ink writing \\
$\mathrm{SLS}$ & Selective laser sintering \\
$\mathrm{AMTs}$ & Additive manufacturing techniques \\
$\mathrm{CAD}$ & Computer-aided design \\
$\mathrm{VP}$ & Vat photopolymerization \\
$\mathrm{MJ}$ & Material jetting \\
$\mathrm{BJ}$ & Binder jetting \\
$\mathrm{ME}$ & Material extrusion \\
$\mathrm{PBF}$ & Powder bed fusion \\
$\mathrm{SL}$ & Sheet lamination \\
$\mathrm{DED}$ & Directed energy deposition \\
$\mathrm{SWCNTs} / \mathrm{SWNTs}$ & Single-walled carbon nanotubes \\
$\mathrm{MWCNTs} / \mathrm{MWNTs}$ & Multiwalled carbon nanotubes \\
$\mathrm{PBT}$ & Polybutylene terephthalate \\
$\mathrm{ABS}$ & Acrylonitrile-butadiene-styrene \\
$\mathrm{PVA} / \mathrm{PVOH}$ & Polyvinylalcohol \\
$\mathrm{Al}{ }_{2} \mathrm{O}_{3}$ & Aluminum oxide \\
$\mathrm{ZrO}$ & Zirconium dioxide \\
$\mathrm{BaTiO}$ & Barium titanate \\
$\mathrm{SiC}$ & Silicon carbide \\
$\mathrm{TiC}$ & Titanium carbide \\
$\mathrm{CBMs}$ & Carbon-based materials \\
$\mathrm{CBMPCs}$ & CBMs-reinforced polymer matrix composites \\
$\mathrm{PLA}$ & Polylactic acid \\
$\mathrm{PC}$ & Polycarbonate \\
& \\
\hline & \\
\hline
\end{tabular}




\begin{tabular}{|ll|}
\hline \multicolumn{1}{|c|}{ Abbreviations } \\
\hline PA & Polyamide \\
STL & Standard tessellation language \\
CAM & Computer-aided manufacturing \\
HBC & Heated build chamber \\
CNT & Carbon nanotubes \\
LDM & Liquid deposition modeling \\
CFRP & Carbon fiber-reinforced polymer composite \\
UV & Ultraviolet \\
VIS & Visible \\
PEO & Poly(ethylene oxide) \\
FDD & Fiber dispensing device \\
PCL & Poly(caprolactone) \\
HA & Hydroxyapatite \\
PANI & Polyaniline \\
PEEK & Polyether ether ketone \\
GO & Graphene oxide \\
PLG & Polylactide-co-glycolide \\
hMSCs & Human mesenchymal stem cells \\
GNP & Graphene nanoplatelet \\
PA-12 & Polyamide-12 \\
RHEC & Rat heart endothelial cells \\
& \\
\hline
\end{tabular}

deviations from the predicted model. Furthermore, conventional manufacturing and machining methods may lead to the wastage of material, the formation of a variety of defect types and geometric inaccuracy in the final product (Ref 9). Therefore, the need to develop new manufacturing techniques that are able to eliminate the limitations of conventional manufacturing techniques led to an interest in additive manufacturing techniques (AMTs). AMTs can be used to fabricate a near net shape composite part with tailored orientation of reinforcement throughout various sections.

AMTs, also known as 3D printing, rapid prototyping, rapid manufacturing, and freeform fabrication, involve the manufacture of an object from 3D model data by the process of joining materials, often layer-by-layer (LBL) on the macro- or microscale (Ref 10-14). To compare the surface characteristics of a fabricated component, a pump bowl fabricated by sand casting and 3D printing is shown in Fig. 1. Since AM processes are based on adding materials to create an object, rather than subtractive manufacture, the level of material waste to obtain the final product is reduced and good geometric accuracy can also be achieved. AM is able to transform a 3D CAD model into the final product without requiring any process planning, extra tooling, and fixtures (Ref 15). Therefore, there is no need to design molds or special fixtures and assemblies to fabricate a complex-shaped part. AM can also reduce the design-manufacturing cycle time, thus decreases the manufacturing cost (Ref 16).

Additive manufacturing can be achieved through a variety of processes, where the method of building and consolidating each layer varies with the process. AM processes can be categorized according to the method of controlling layer fabrication, i.e., variable mass, variable energy, and variable mass and energy process (Ref 17). In the variable mass process, layer formation controls mass while keeping the energy constant, whereas, in a variable energy process, selective bonding of a constant mass takes place by variable energy. Both mass and energy vary in variable mass and energy processes. Some AM processes use photopolymer liquids as starting materials (Ref 18), while others use powders (Ref 19), filament/paste (Ref 20) or solid sheets (Ref 21) as the starting material. The working principle of the AM process varies with the state of starting material. An ASTM committee F42 divided $\mathrm{AM}$ processes into seven main categories, namely.

1. VAT photopolymerization (VP),

2. Material jetting (MJ),

3. Binder jetting (BJ),

4. Material extrusion (ME),

5. Powder bed fusion (PBF),

6. Sheet lamination (SL),

7. Directed energy deposition (DED).

VP, BJ, PBF, and SL can be grouped into variable mass processes, whereas $\mathrm{MJ}$ and $\mathrm{ME}$ can be grouped into variable energy processes. Both mass and energy vary in DED. These
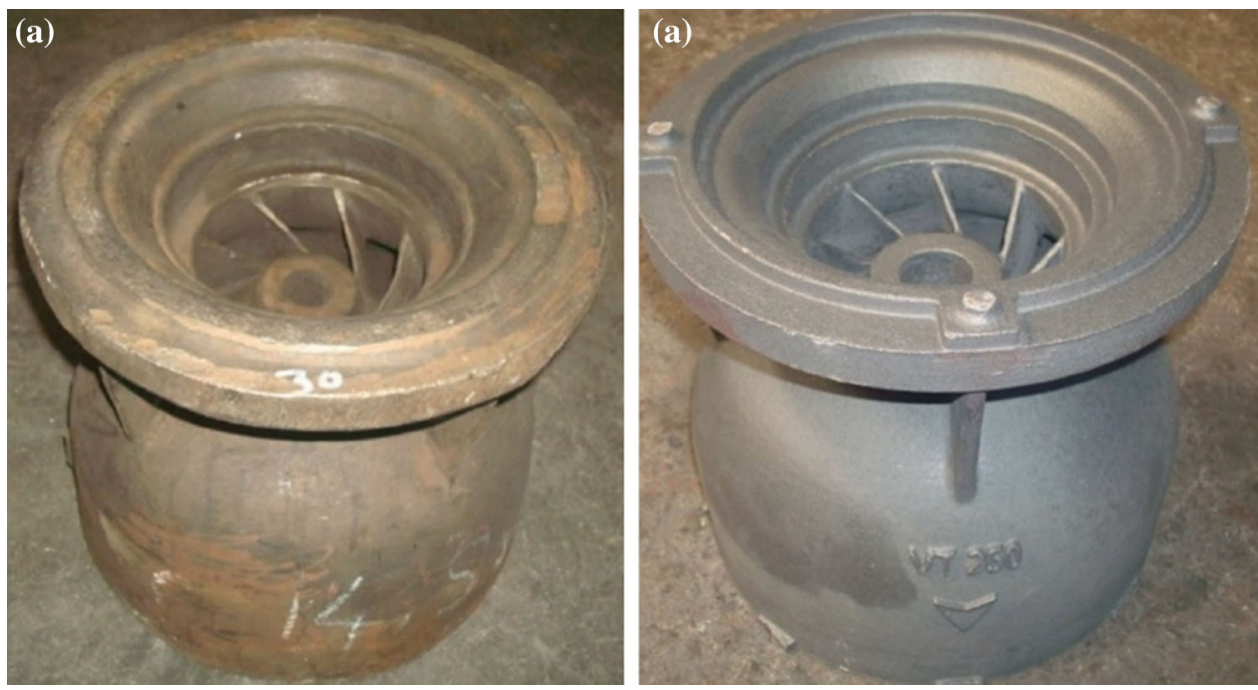

Fig. 1 A pump bowl fabricated by (a) conventional sand casting and (b) 3D printing 
seven categories can further be classified into several AM technologies which are shown in Table 1.

The broad classification of AM technologies is presented in Table 1, but some AM technologies adopted for the manufacturing of composites can differ somewhat. Table 2 shows examples of additively manufactured composites and their adopted AM technology.

From Table 2, it can be observed that AMTs can be used to manufacture composites with a variety of reinforcement geometries. The properties of the reinforcement play a vital role in determining the effective properties of final composites. Researchers have worked on various reinforcements, but the use of carbon-based reinforcements is increasing at a significant rate. The properties of carbon-based materials (CBMs) depend upon the local bonding of carbon atoms. Thus, their properties

Table 1 Main AM processes and technologies

\begin{tabular}{ll}
\hline AM process & \multicolumn{1}{c}{ AM technologies } \\
\hline VAT photopolymeriza- & 1. Stereolithography (SLA) \\
tion & 2. Direct light processing (DLP) \\
3. Continuous DLP (CDLP) & 1. Material jetting \\
Material jetting & 2. Nanoparticle jetting \\
& 3. Drop-on-demand (DOD) \\
Binder jetting & 1. Binder jetting \\
Material extrusion & 1. Fused deposition modeling (FDM) \\
Powder bed fusion & 1. Selective laser sintering (SLS) \\
& 2. Selective laser melting (SLM) \\
3. Direct metal laser sintering (DMLS) & 4. Electron beam melting (EBM) \\
& 5. Multijet fusion (MJF) \\
6. Selective heat sintering (SHS) & 1. Ultrasonic additive manufacturing \\
(UAM) & 2. Laminated object manufacturing (LOM) \\
1. Laser-engineered net shape (LENS) & 2. Electron beam additive manufacture \\
& (EBAM) \\
\hline &
\end{tabular}

differ greatly although they are made up of the same element, and examples of CBMs are shown in Fig. 2.

CBMs possess excellent electronic (Ref 37), mechanical (Ref 38), thermal (Ref 39), optical (Ref 40), hydrogen sorption (Ref 41), and magnetic properties (Ref 42). Thus, these materials are potential reinforcements in polymer matrix composites. The additive manufacturing of CBMs-reinforced polymer matrix composites (CBMPCs) can be realized by several methods, which are discussed in the next section. Factors that need to be considered for the additive manufacturing of CBMPCs include the viscosity of filler/matrix mixture and bonding between matrix and filler (Ref 43). Thermosetting as well as thermoplastic polymer materials, such as epoxy resin (Ref 44), polylactic acid (PLA) (Ref 45), polycarbonate (PC) (Ref 46), polyamide (PA) and many others (Ref 47), can be processed by additive manufacturing.

This review focuses on the AM of carbon materials-based small-scale structures and fiber-reinforced polymer composites for engineering applications. Although few reviews are already present on the AM of carbon fillers-based polymer composites, they are mainly focused on the fused deposition modeling process. In this review, other widely adopted AM processes for carbon fillers-based polymer composites are also considered. In the first section, examples of AM processes adopted for the fabrication of CBMPCs are discussed. Then, some additively manufactured CBMPCs with their properties and performances are examined.

\section{Main AM Technologies Adopted for the Fabri- cation of CBMPCs}

Several AM technologies have been used for the fabrication of CBMPCs, where each technology has its advantages and disadvantages. The main AM technologies adopted for the fabrication of CBMPCs are discussed in this section with their advantages and disadvantages summarized in Table 3.

Table 2 Examples of additively manufactured composite materials and adopted process

\begin{tabular}{|c|c|c|}
\hline Composite material & AM technology & References \\
\hline Multiwalled carbon nanotubes (MWCNTs)/polylactic acid (PLA) & Liquid deposition modeling & Ref 22 \\
\hline Graphene/polybutylene terephthalate (PBT) & Fused deposition modeling & Ref 23 \\
\hline Graphene/acrylonitrile-butadiene-styrene (ABS) & Fused deposition modeling & Ref 24 \\
\hline Carbon nanotubes/acrylonitrile-butadiene-styrene (ABS) & Fused deposition modeling & Ref 25 \\
\hline Carbon nanotubes/polyvinylalcohol (PVOH) & Fused deposition modeling & Ref 26 \\
\hline Carbon fibers/acrylonitrile-butadiene-styrene (ABS) & Fused deposition modeling & Ref 16 \\
\hline $\mathrm{Al}_{2} \mathrm{O}_{3} / \mathrm{ZrO}_{2}$ & Stereolithography & Ref 27 \\
\hline $\mathrm{BaTiO}_{3} / \mathrm{ABS}$ & Fused deposition modeling & Ref 28 \\
\hline $\mathrm{Al}_{2} \mathrm{O}_{3} /$ urethane diacrylate and Isobornyl acrylate & 3D magnetic printing (SLA based) & Ref 29 \\
\hline Optical fiber/aluminum & Ultrasonic additive manufacturing & Ref 30 \\
\hline Carbon and $\mathrm{SiC}$ whiskers/epoxy cellular composite & Fused deposition modeling & Ref 31 \\
\hline Acrylamide gel/epoxy & Stereolithography & Ref 32 \\
\hline SWCNT/alginate & Multinozzle biopolymer deposition & Ref 33 \\
\hline Copper wire/BendLay polymer & Fiber Encapsulation Additive Manufacturing & Ref 34 \\
\hline VeroWhitePlus/TangoBlackPlus (both are acrylic-based photopolymer resins) & Dual material jetting (SLA based) & Ref 35 \\
\hline TiC/Ti6Al4V (a titanium alloy) & Laser melting deposition & Ref 36 \\
\hline
\end{tabular}


(a)

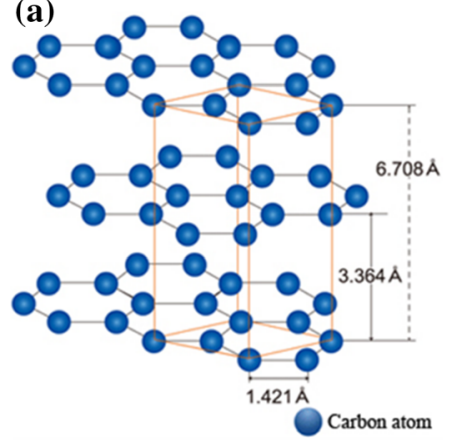

(c)

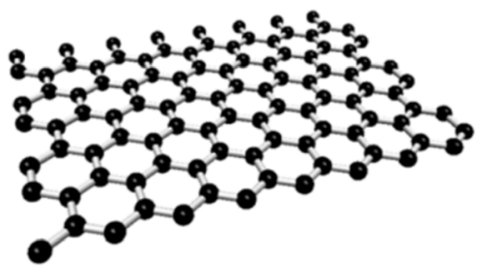

(b)
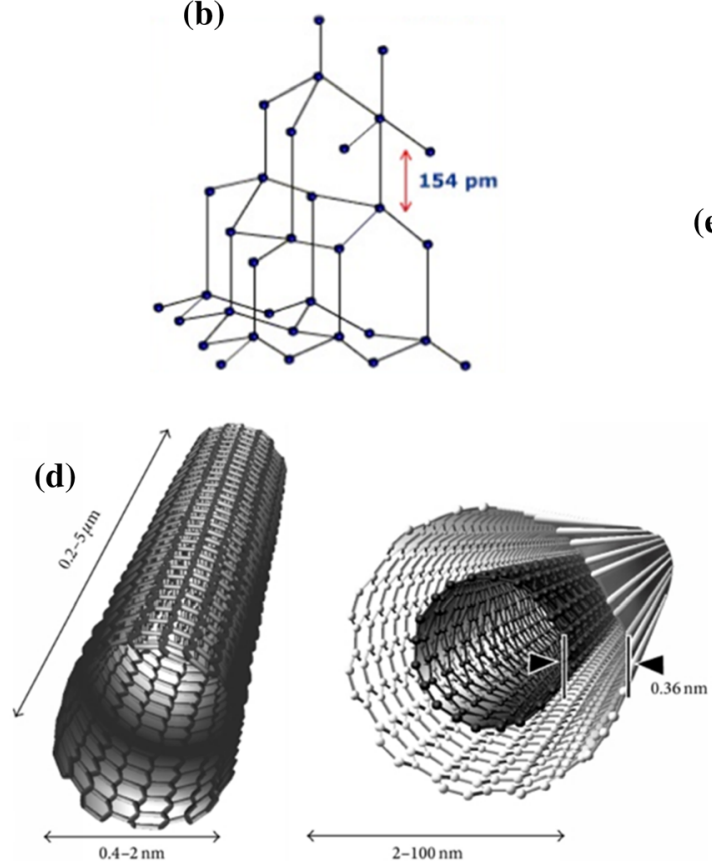

(e)

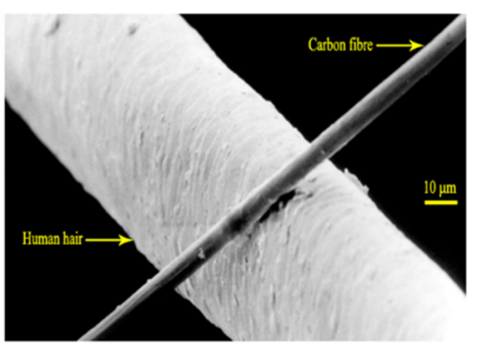

Fig. 2 Carbon-based materials, (a) graphite, (b) diamond, (c) single-layer graphene, (d) single-walled and multi-walled carbon nanotubes, and (e) carbon fibers

\subsection{Fused Deposition Modeling (FDM)}

Among the various AM technologies shown in Table 3, FDM is used most widely for the fabrication of polymer matrix composites due to minimal material wastage, low cost, and ease of material change (Ref 16). The process involves a computercontrolled material extrusion and deposition process. The standard tessellation language (STL) file generated by CAD software is sliced into horizontal layers, and these horizontal layers are built up layer-by-layer onto a platform, as shown in Fig. 3. In this process, a geometric model is initially generated using CAD software. The geometric model is then translated into an STL file which is exported to a CAM unit. The model is examined for optimum deposition direction, and the STL file is mathematically sliced into horizontal layers. Support structures are then generated. The created tool paths are reviewed, and the data are sent to the FDM system (Ref 52).

FDM system includes a material feed mechanism, print head, liquefier, gantry and builds surfaces as key elements (Ref 53). In FDM systems, filaments of the desired material are fed to an extrusion nozzle from a spool of materials. The heated extrusion nozzle melts the filaments, and the melted materials are extruded and deposited over a flat base called the build platform or table. The motion of the nozzle, and the table, is controlled by a computer along both horizontal and vertical directions. The extruded materials are deposited layer-by-layer, and the diameter of the nozzle defines the resolution of printing. These layers then fuse and solidify into the final part. Printing parameters such as raster angle, raster width, printing orientation, model build temperature, air gap, and layer thickness can be altered to control the quality of printed parts (Ref 54, 55).

To fabricate particulate and chopped fiber-reinforced polymer composites, reinforcements are mixed with thermoplastic filaments before their loading in the printer (Ref 4). Some printers also use multi-printer heads to fabricate composites (Ref 56). However, continuous fibers-reinforced composites cannot be fabricated by simply mixing the fibers with thermoplastic filaments. There is currently no standard and robust technique to fabricate continuous fibers-reinforced polymer composites. To fabricate continuous fiber composites, an in-nozzle impregnation method is generally used (Ref 4, 57), as shown in Fig. 2(b). Figure 4 shows a schematic representation of FDM and in-nozzle impregnation setup with some 3Dprinted composites.

The in-nozzle impregnation method utilizes a conical nozzle to print the composites in which continuous fiber and polymer filament are supplied separately and mixed uniformly by heating the polymer filament to its glass transition temperature (Ref 57). Continuous fiber-reinforced polymers stick to the panel during printing and provides a sufficient traction force to the incoming mixture (Ref 57). This traction force pulls polymer-cladded fiber out of the nozzle and builds the part layer-by-layer.

Several carbon-based particulate and fiber-reinforced polymer composites have been fabricated by the FDM approach. Wei et al. (Ref 58) fabricated graphene/ABS composite by FDM, and it was found that the temperature of the nozzle, heated build chamber (HBC), heated build platform, and the glass transition temperature of the printing materials affect the print quality. The temperature of the heated build chamber should be sufficiently high to soften the matrix and low enough so that it should not degrade the polymer ingredients. A high concentration of filler may lead to jamming of the nozzle. In addition, the incorporation of hard carbonaceous reinforcements in polymer filaments may lead to the wear of nozzle, which subsequently reduces the resolution of printing ( $\operatorname{Ref} 23$ ). This also degrades the esthetics and the functional properties of the composites. Thus, designing the nozzle to print abrasive materials and prevent jamming of the nozzle at a high concentration of filler should be taken into consideration (Ref $23,58)$. 


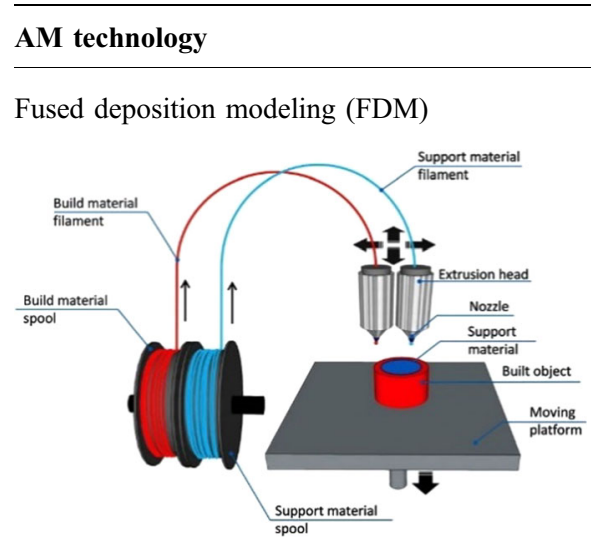

(Ref 48)

Stereolithography (SLA)

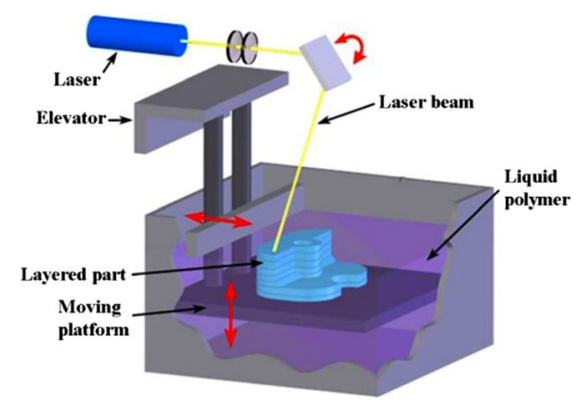

(Ref 49)

Direct ink writing (DIW)

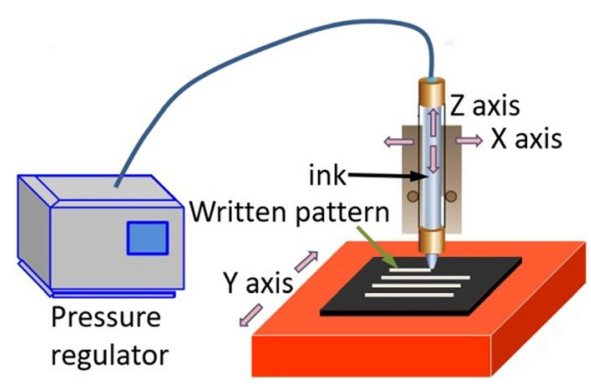

(Ref 50)

Selective laser sintering (SLS)

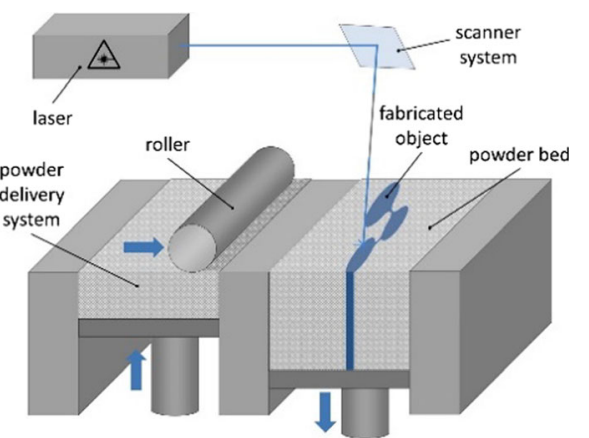

Low cost

Clean and easy process

A wide range of thermoplastic

materials can be used

Compact size of setup makes it appropriate for remote manufacturing abilities

High accuracy

Large-sized parts can be manufactured Printing resolution is high $(50-100 \mu \mathrm{m})$ A considerable degree of design freedom Limited finishing required in printed parts

Excellent surface finish of fabricated part (i.e., surface roughness of about tens of microns)

Room temperature processing environment
Disadvantages

Removing support material from the model may lead to damage of model

Lines between the layers are generally observed in the fabricated part

High porosity

Weak link between the different layers. Thus, it may lead to delamination of layers upon loading Chance of blockage of nozzle

Limited to UV-curable resins

UV-curable resins generally brittle and low strength

SLA system is expensive

Process is slow

Fabricated parts are fragile

Printing resolution is low. $(200-400 \mu \mathrm{m})$
Less reliance for supports

Complex parts can be fabricated with ease A wide range of materials can be printed Fast process
Fabricated parts are generally porous Rough surface of fabricated parts

Thermal distortion may occur which can cause warping and shrinking of fabricated parts

$(\operatorname{Ref} 51)$ 

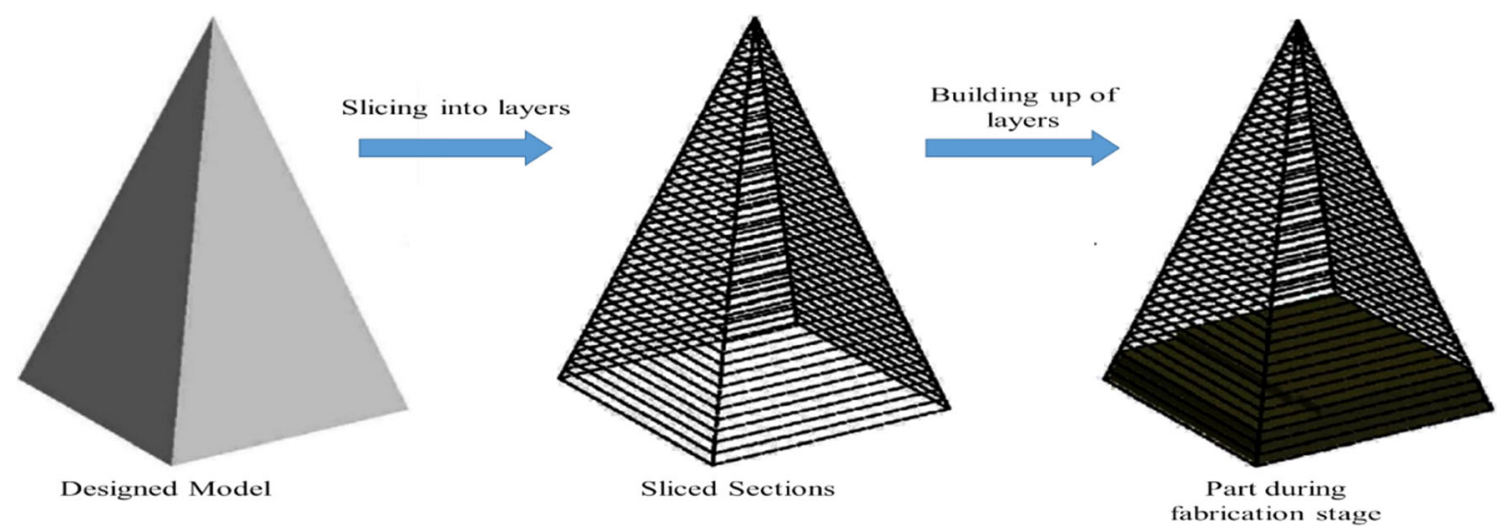

Fig. 3 Flowchart showing building up of layers. Reprinted from Ref. 1 with permission from Elsevier, Copyright 2015
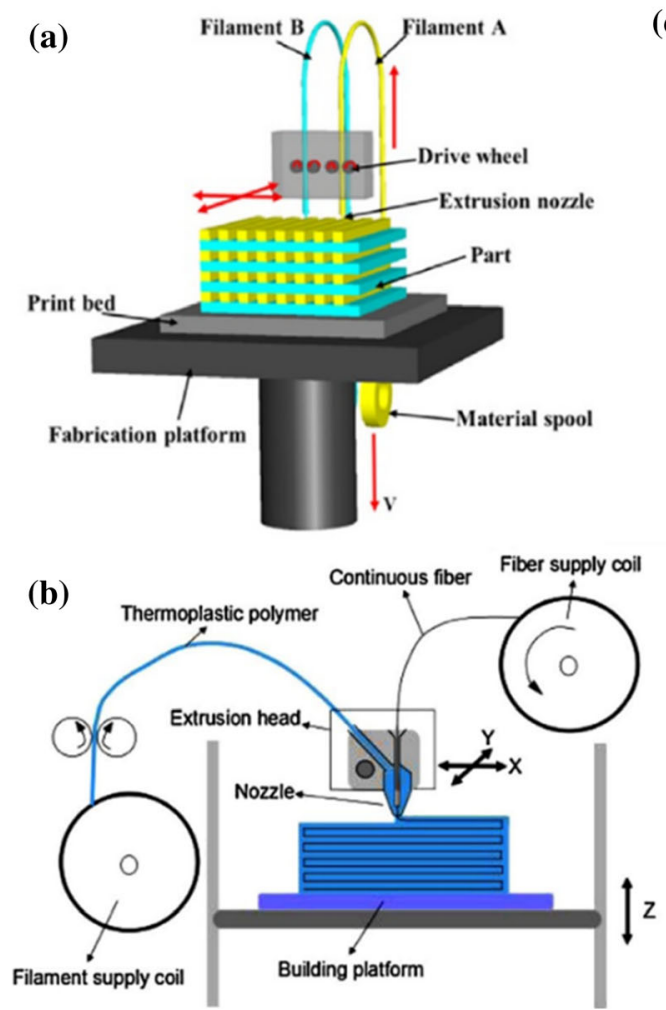

(c)
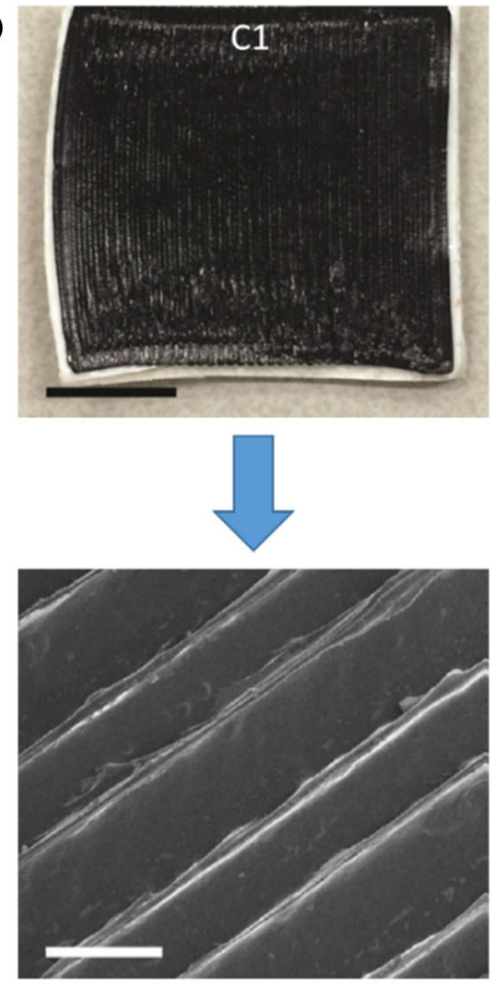
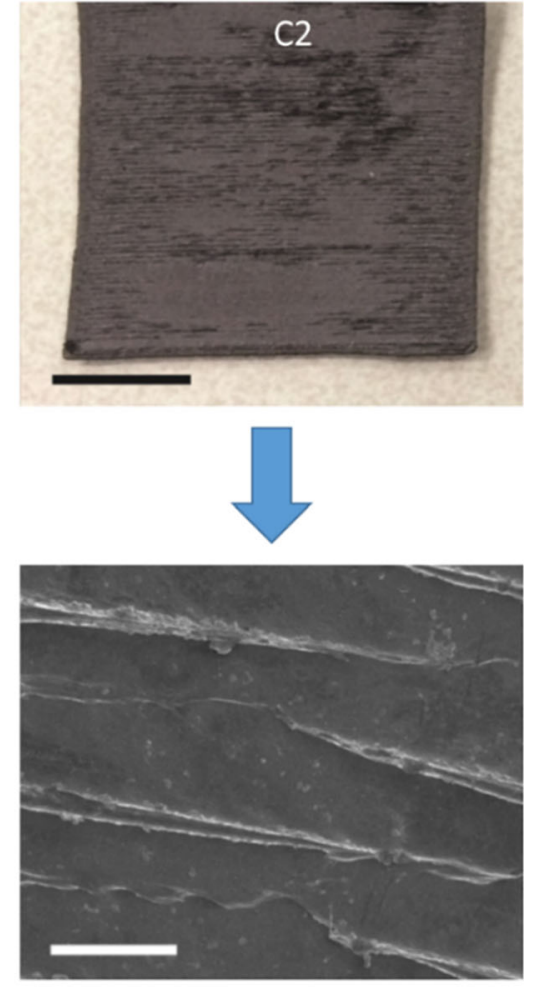

Fig. 4 Schematic of a (a) typical FDM setup and (b) in-nozzle impregnation, (c) 3D-printed monolayer of polybutylene terephthalate (PBT)/ CNT composite (C1) and PBT/graphene composite (C2) with their SEM images. Panel (a) is reprinted from Ref 54 with permission from Elsevier, Copyright 2016. Panel (b) is reprinted from Ref 57 with permission from Elsevier, Copyright 2016. Panel (c) is reprinted from Ref 23

Gnanasekaran et al. (Ref 23) fabricated CNT and graphenebased polybutylene terephthalate (PBT) composites. They observed a ridge pattern between the different layers since the printing nozzle touched the previously printed layers leading to material flow, although reducing the nozzle temperature may eliminate this problem, but it leads to an increase in viscosity which increases the tendency of nozzle jam. Thus, the nozzle temperature must be chosen to balance these two phenomena. Carbon nanotubes have also been used in combination with acrylonitrile-butadiene-styrene (ABS) (Ref 25, 59) and polyvinylalcohol (PVOH) (Ref 26). Prashantha and Roger (Ref 60) fabricated graphene/PLA composites by FDM and found that the time between the deposition of continuous filaments affects the porosity of fabricated composites. Less porosity was observed when the deposition time was shorter, and this was due to better inter-joining and interaction between contiguous filaments. This low porosity was due to the lowtemperature difference between already printed layers and the layer being printed. Better inter-joining can also be obtained by increasing the heating time using hot air (Ref 61); however, this may result in a disruption of morphology. Sweeney et al. (Ref 62) fabricated CNT/PLA films by FDM and showed that local microwave heating can be used to weld the printed filaments which reduce the probability of delamination of filaments during in-service conditions; this use of local heating does not affect the morphology. Dul et al. (Ref 24) incorporated 4 wt.\% graphene nanoplatelets in an ABS matrix to form a nanocomposite by FDM where they found that the microstructure of the 
3D-printed parts changes with build orientation of the layers. The mechanical properties also vary with the change in build orientation. A slight variant of FDM, termed liquid deposition modeling (LDM), has also been developed which eliminates the heating and melting of filament in the nozzle (Ref 22). The part can be printed directly from the liquid precursors. Postiglione et al. (Ref 22) fabricated MWCNT/PLA composites by LDM, and it is cost-effective as compared to the typical FDM process.

Carbon fiber-reinforced polymer composites have also been fabricated by FDM. Ning et al. (Ref 16) fabricated short carbon fiber-reinforced polymer composite by FDM. They showed that porosity increases when the content of carbon fiber increased to $10 \mathrm{wt} . \%$, and this was due to inconsistent fusion of layers because of different carbon fiber distributions in the filament. Ning et al. (Ref 63) also fabricated carbon fiber-reinforced polymer composite (CFRP) by FDM with 5 wt.\% of chopped carbon fibers in an ABS matrix. They found that the process parameters such as raster angle, nozzle temperature, infill speed, and layer thickness affect the mechanical properties of the fabricated composite. Tekinalp et al. (Ref 64) showed that FDM yields a high orientation of short carbon fibers in the printing direction $(\sim 91.5 \%)$, whereas compression molding leads to a low orientation of fibers in a specific direction.

Continuous carbon fiber-reinforced polymer composites can also be fabricated using FDM. Li et al. (Ref 57) fabricated continuous carbon fiber/PLA composites and showed that continuous fibers can be printed along a curved path. The nozzle flattens the printed carbon fiber/PLA composite which results in good fusion. However, there is a slight variation in the width of PLA resin between carbon fibers. Hatch spacing (central distance between two adjacent lines) also affects the quality of printing in continuous carbon fiber-reinforced polymer composites using FDM (Ref 57). Decreasing the hatch spacing causes the overlapping of layers, and increasing it may lead to the low resolution of printing.

The continuous carbon fiber-reinforced thermoplastic polymers had been fabricated via FDM. The FDM-based platform could be modified to print thermosetting polymers-based composites. In a research work, Hao et al. (Ref 65) slightly modified FDM setup to print continuous carbon fiber-reinforced epoxy composites. In their modified setup, the fiber bundle from the supply coil firstly proceeded through the epoxy spool before entering the printing head. A three-rod mechanism was used to control the content of epoxy on the fiber bundle. After printing, the composite was cured at a high temperature.

FDM is a widely used process in the fabrication of polymer composites. There are several other techniques that are employed for the fabrication of polymer composites which are discussed in the subsequent sections.

\subsection{Stereolithography}

Stereolithography (SLA) takes less time for fabrication as compared to other conventional prototype techniques such as FDM and SLS (Ref 66). The process utilizes a UV or VIS light source to construct an object by curing a thin layer of liquid resin via photopolymerization layer-by-layer (Ref 67). Commercial SLA was developed in 1986 by 3D systems (Ref 68), and to fabricate a component using the SLA technique, initial steps such as the generation of STL file and the mathematical slicing of the model are carried out in the same way as in FDM process. After slicing, the data are transferred to an SLA apparatus and the component is fabricated by spatially controlled solidification (by photopolymerization) of liquid resin. The surface of the resin is illuminated at specific locations by a computer-controlled laser beam (Fig. 5a) or a digital light projector (Fig. 5b). This results in solidification of the resin to a certain depth, and it adheres to the support platform. The platform is then moved away from the surface with a depth slightly smaller than the curing depth, and the process is repeated until the whole component is generated. The excess resin is then washed off to obtain green structure which is postcured, generally by ultraviolet light to enhance its mechanical properties.

Several carbon-based polymer matrix composites have been fabricated by the SLA technique. Kalsoom et al. (Ref 69) fabricated a microdiamond-reinforced acrylate resin composite using the SLA technique. SEM images of the fabricated composite are shown in Fig. 5(c) and (d), and they found that adhesion of the printed layer to the printer platform depends upon the concentration of filler; it was noted that the higher the filler concentration, the lower the adhesion. Furthermore, the concentration of filler also affected the curing time. It can be observed from Fig. 5(c) and (d) that the gap between diamond particles was significant at $10 \mathrm{wt} . \%$ filler, whereas it reduced to a greater extent at $30 \mathrm{wt} . \%$, which affected the curing time.

Chiappone et al. (Ref 70) fabricated graphene oxide (GO)reinforced $\mathrm{PEO}$-acrylates composites and found that an increase in the concentration of filler led to an increase in curing time by UV-Vis light. A photoinitiator working under the light of a longer wavelength can also be used to ensure better curing, and the cure depth of resin also depends upon the time of exposure of UV-Vis light (Ref 71). However, incorporating a filler also affects the curing characteristics of the resin. They also compared the curing characteristics of the composite film under thermal treatment and UV-Vis light. Thus, parameters such as exposure time and wavelength of light should be optimized to achieve uniform curing extents throughout the layer. Sandoval et al. (Ref 72) fabricated MWCNTs-reinforced epoxy resin composites by SLA. They found that a long exposure time leads to the generation of internal stresses during curing which may cause the deformation ("curling") of layers, as can be observed in Fig. 5(f). Figure 5(e) shows a specimen of pure epoxy resin which does not exhibit any part curling. Incorporation of MWCNTs also leads to a significant increase in the surface tension of the MWCNTs/epoxy mixture which also causes deformation of layers and overall fabricated part.

SLA generally leads to a random orientation of the particulate filler without controlling any orientation (Ref 74, 75). However, the orientation of continuous fibers can be controlled in SLA (Ref 76). To reinforce continuous fibers, a fiber dispensing device (FDD) is generally used which is controlled electronically by a motion controller regulated by a computer (Ref 77). FDD dispenses fiber tows on the surface of the base member. There is a relative motion between base and FDD which enables FDD to dispense multiple fiber tows at different locations. Reinforcing carbon fibers in polymers increases its opacity toward UV light (Ref 78). Thus, it is difficult to cure resin nearby fiber and embedded in between the fiber filaments.

\subsection{Direct Ink Writing}

Direct ink writing (DIW) is an alternative to FDM which prints viscoelastic materials under ambient conditions (Ref 79). 
(a)

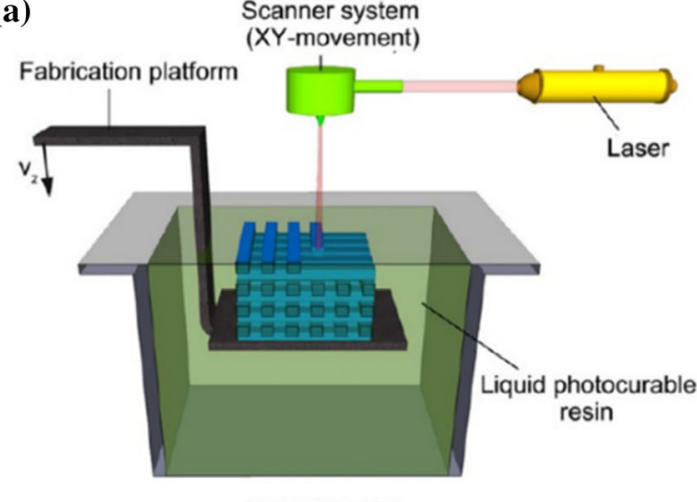

BOTTOM-UP

(c)

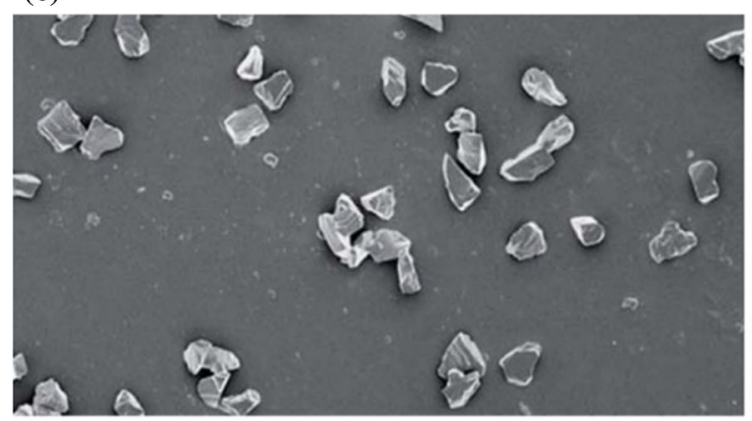

(e)

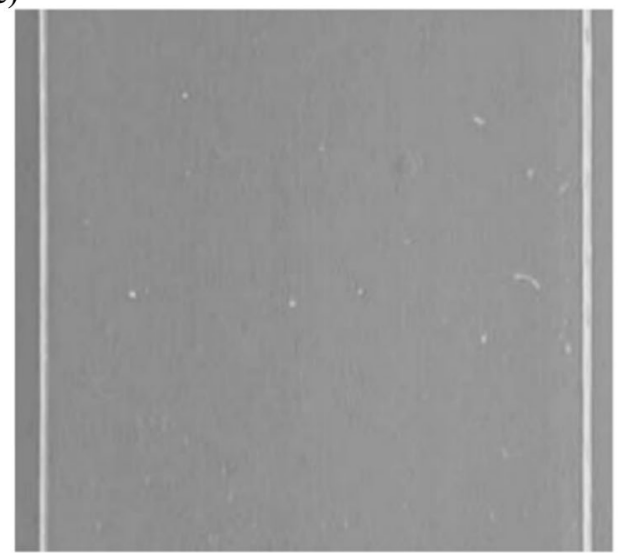

(b)

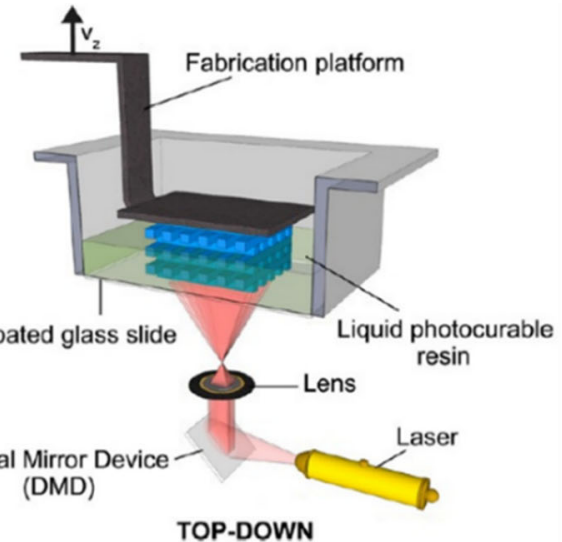

(d)

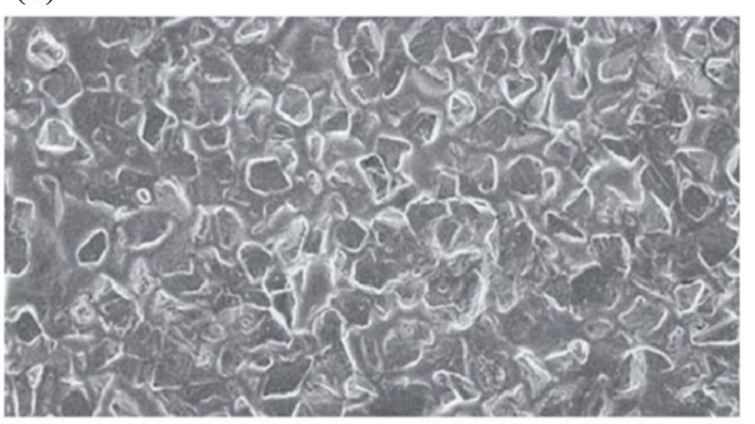

(f)

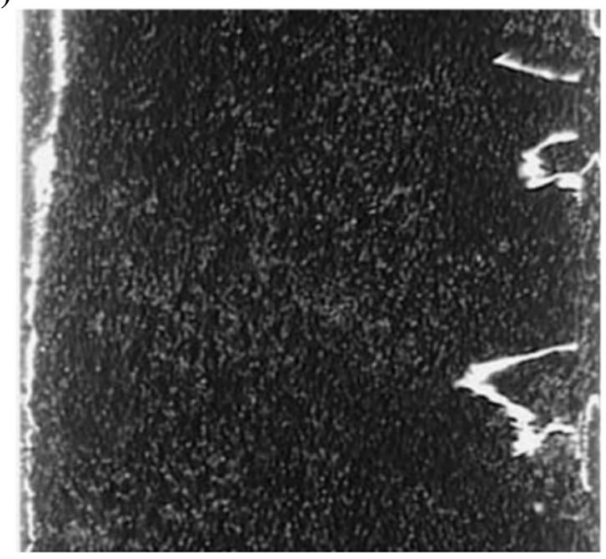

Fig. 5 Schematic showing (a) bottom-up (computer-controlled laser beam) and (b) top-down (digital light projector) approach for stereolithography. SEM images showing microdiamond-reinforced acrylate resin composite fabricated by DLP-based SLA technique when the weight percentage of microdiamond is (c) 10\% and (d) 30\%. SEM images showing (e) epoxy resin specimen and MWCNTs-reinforced epoxy resin composite fabricated by SLA. Panels (a) and (b) are reprinted from (Ref 73) with permission from Elsevier, Copyright 2012. Panels (c) and (d) are republished with permission of Royal Society of Chemistry, from "A 3D printable diamond polymer composite: A novel material for fabrication of low cost thermally conducting devices," U. Kalsoom, A. Peristyy, P. Nesterenko, B. Paull, Vol 6 (44), Copyright 2016 (Ref 69); permission conveyed through Copyright Clearance Center, Inc. Panels (e) and (f) are reprinted by permission from Springer Nature: Springer Nature, Journal of Materials Science (Ref 72), Copyright 2006

In DIW, a controlled flow rate of ink (material mixture in the liquid phase) is dispensed from a fine deposition nozzle using a pressurized syringe and is deposited in a similar fashion to FDM. Its setup is almost the same as the setup for FDM, as can be observed from Fig. 6. Post-curing of fabricated parts is required to achieve appropriate mechanical properties. 3D structures with high aspect ratio, continuous solids or spanning features can be constructed by direct ink writing.

DIW techniques are generally divided into filamentarybased (e.g., robocasting and micro-open writing) and droplet- based (e.g., ink-jet printing and hot melt printing) approaches (Ref 80). The design of ink and its rheology are crucial parameters. To design appropriate inks, its viscoelastic response and setting capability are kept in mind. The viscoelastic response determines its "flow" characteristics through the deposition nozzle, and an ideal ink should possess shear thinning behavior with moderate yield stress (Ref 81) to facilitate extrusion under a shear force. It is to be set time (pseudoplastic to dilatant recovery) that should be low to facilitate shape retention of deposited features. Compressive 


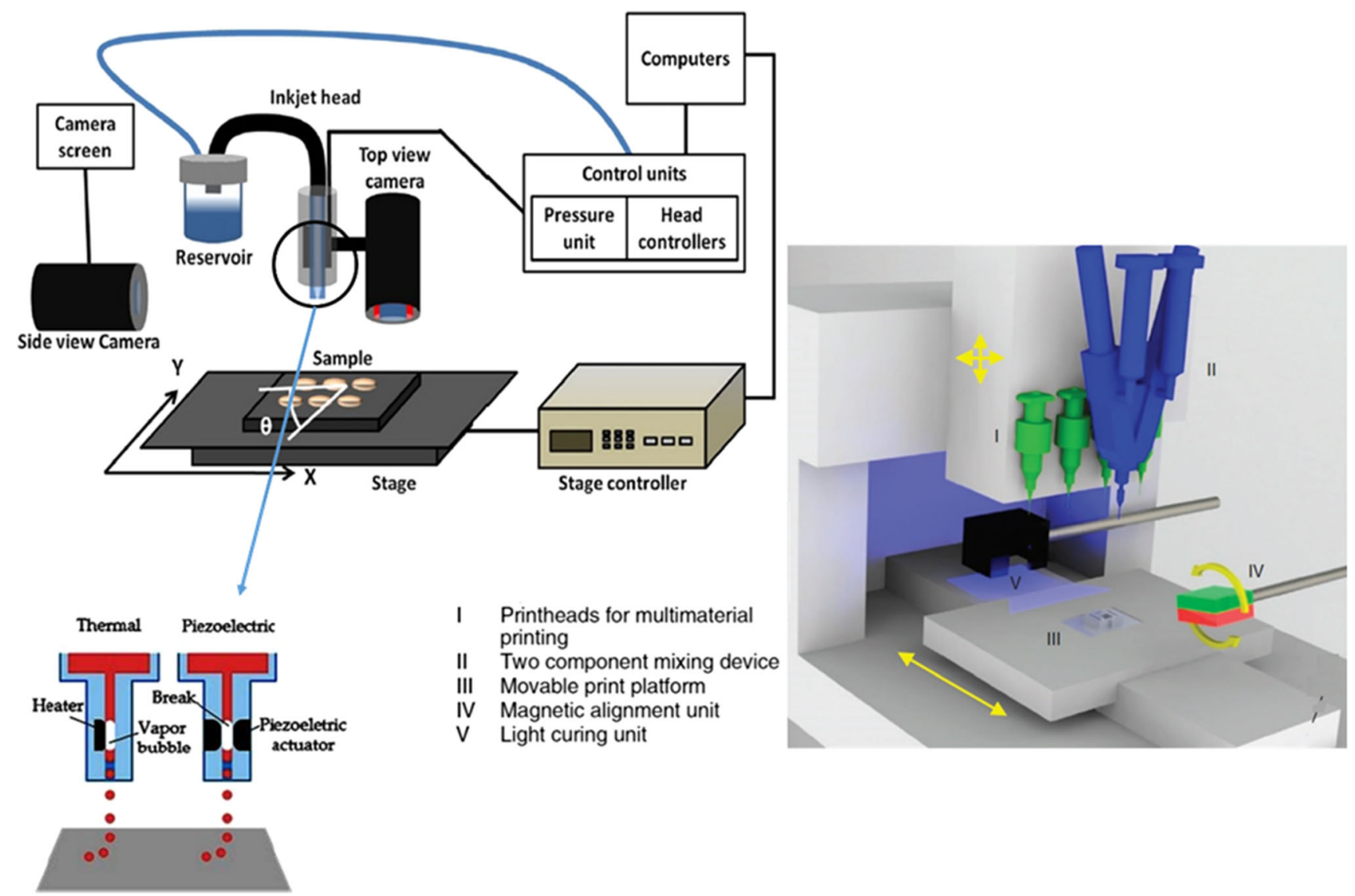

Fig. 6 Schematic showing setup for direct ink writing. The top left image is reprinted from (Ref 83). (C) IOP Publishing. Reproduced with permission. All rights reserved. The right and bottom left images are reproduced from (Ref 84) and (Ref 85), respectively

stresses are generally induced from capillary tension (Ref 82). Thus, the volume fraction of colloid should be high to minimize shrinkage due to drying after generation of the part. The generated particle network should be able to resist the induced compressive stresses. But high colloidal volume fraction may affect the viscoelastic response and set time of the ink. Thus, the optimum of the three parameters is required.

Dorj et al. (Ref 86) fabricated a nanocomposite bone scaffold of ionically modified carbon nanotubes (imCNTs)reinforced poly(caprolactone) (PCL) - hydroxyapatite (HA) by robocasting. They found that the incorporation of $0.2 \mathrm{wt} . \%$ of imCNTs in PCL-HA has good flow characteristics and significantly improved the elastic modulus and compressive strength of scaffolds. Lewicki et al. (Ref 81) fabricated mesostructurally ordered carbon fiber and silica/polymer composites by DIW. Their investigation showed that a high degree of fiber alignment could be achieved using DIW, as shown in Fig. 7(a), which outperformed the mechanical and electrical properties of the equivalently filled CFRP composites with randomly oriented carbon fibers. The flow characteristics of the fabricated composite are shown in Fig. 7(b), and it can be observed that the base epoxy resin acted as Newtonian fluid, whereas the reinforcement of silica and carbon fiber changed its behavior to the non-Newtonian fluid. This change in flow properties ultimately affects the DIW processing parameters.

Compton et al. (Ref 87) reinforced graphene in epoxy at a range of 10, 15, and 20 weight percentages of graphene. They found that rheology and shear thinning behavior of prepared ink vary with the percentage of filler (Ref as shown in Fig. 7(c) and (d)) which had a high effect on the effectiveness of DIW. CNT-reinforced poly(3,4-ethylenedioxythiophene) composites fabricated by piezoelectric inkjet printing revealed that the percentage of filler has a significant effect on the thickness of layers (Ref 88). Cong et al. (Ref 89) prepared a graphene/PANI paper with good cycling stability for supercapacitors using inkjet printing.

\subsection{Selective Laser Sintering}

Selective laser sintering (SLS) comes under the category of powder bed fusion, and it can be used to generate complex 3D parts by solidifying successive layers of required material (in the form of powder) on the top of each other (Ref 19). Solidification is carried out on selective areas, based on the CAD model, and the final part is obtained by fusing or sintering successive layers. The thermal energy required for fusion/ sintering is supplied through a laser beam that scans each layer based on the CAD model, as shown in Fig. 8(a). After sintering one layer, a powder deposition system then deposits a thin layer of powder on the top of the first layer, and the process is continued until the whole part is generated, as shown in Fig. 8(b). SLS can build complex structures without the requirement of any tooling. The majority of the polymer matrix composites fabricated by SLS is based on the thermoplastic polymer matrix, although some researchers have also attempted to use thermosetting polymer matrices (Ref 90).

Zhu et al. (Ref 90) used PA12-coated carbon fibers to reinforce an epoxy matrix to form a ternary composite by SLS. 
(a)

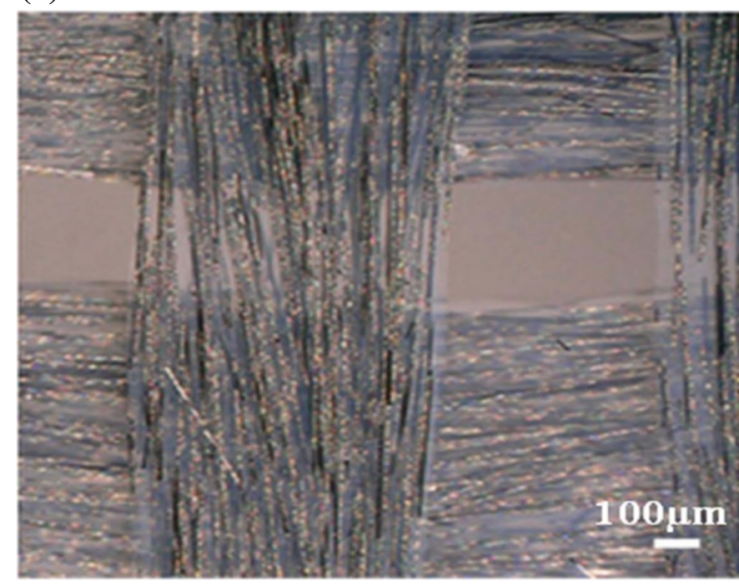

(c)

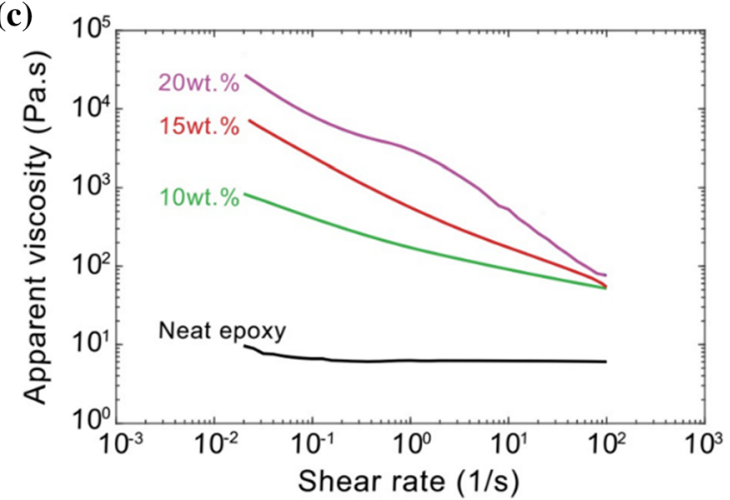

(b)
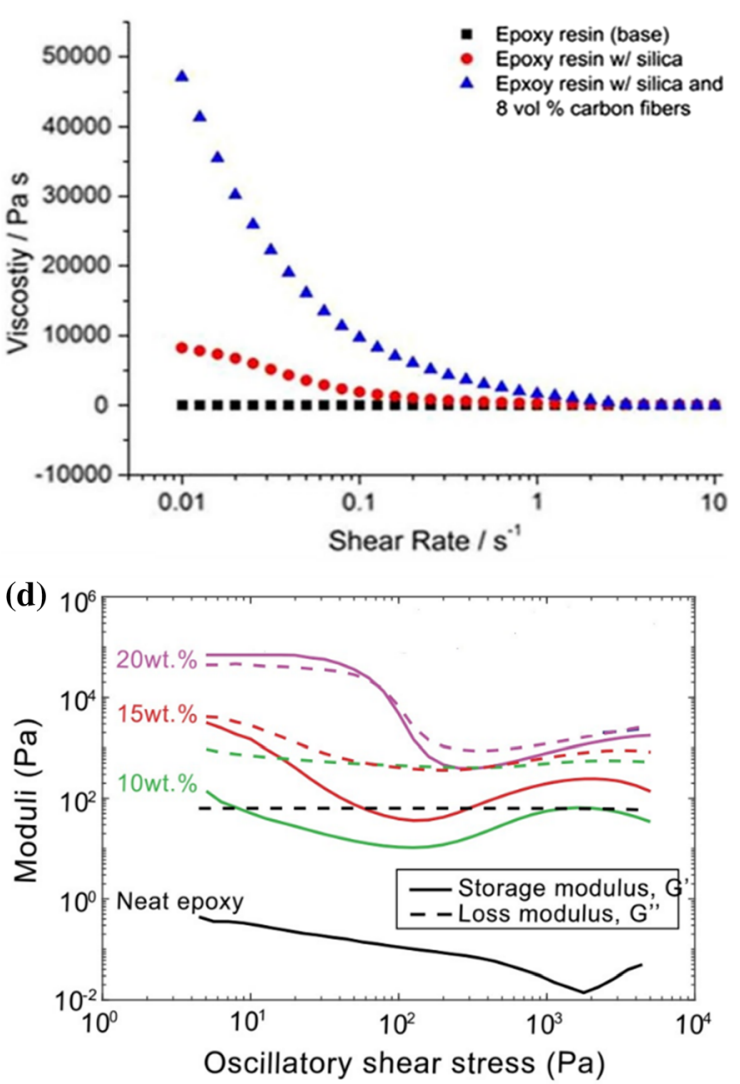

Fig. 7 Panel A; (a, b): (a) highly aligned carbon fibers in epoxy matrix, (b) experimentally derived shear rate-dependent viscosity of epoxy resin and carbon fiber-reinforced epoxy resin at $30^{\circ} \mathrm{C}$. Panel B; (c, d): (c) apparent viscosity and (d) storage and loss moduli for neat epoxy and graphene-reinforced epoxy composite having different weight percentages of graphene. Panel A is reproduced from (Ref 81). Panel B is reprinted by permission from Springer Nature: Springer Nature, JOM: The Journal of The Minerals, Metals \& Materials Society (Ref 87), Copyright 2017

They found that up to $31 \%$ volume fraction of carbon fibers with a good dispersion of carbon fibers in the epoxy matrix can be obtained by SLS. The incorporation of carbon fibers in a polyamide- 12 matrix decreases its melting temperature which in turn reduces the SLS part bed temperature (Ref 91). This leads to lower energy for sintering and less thermal degradation occurs during the sintering process. Laser power and scan speed are important process parameters in SLS (Ref 92), and a high laser power may lead to the pyrolysis of the polymer matrix which manifests itself in the form of porosity. High scan speed results in inadequate sintering of powder, and if the polymer powder does not melt adequately, it results in porosity due to the improper fusion of powder particles (Ref 93). Parameters such as laser scan spacing, powder layer thickness, and the roller speed also affect the quality of a part manufactured through SLS (Ref 94).

Discontinuous carbon fiber-reinforced polymer composites could be fabricated via SLS. However, the fiber length is limited by powder structure. Thus, impossible objects had developed a process that is based on the sheet lamination of the composites (Ref 95). A carbon fiber tissue paper-like sheets are taken, and binder is transferred to them after which they are stacked. Then, the sheets are pressed together to form the composite. Thus, very complex shaped could be fabricated in this way. But only external geometry can be optimized to high precision, whereas its ability to optimize the fiber orientation is not very high (Ref 95).

Particulate reinforced composites could also be fabricated by SLS. In a research work, Yuan et al. (Ref 93) fabricated MWCNT/polymer composites by SLS and showed that segregated structures can be fabricated using the SLS technique. The time of coalescence of the polymer and carbon reinforcement is important in SLS since it influences specific parameters such as time between the deposition of layers and the number of exposures required (Ref 98). Chen et al. (Ref 98) observed an approximately 10- to 20-s delay in the onset of coalescence of graphene/PEEK nanocomposite as compared to plain PEEK, as shown in Fig. 8(c). The surface morphology was observed to change with the incorporation of graphene, as shown in Fig. 8(d) and (e). The morphology of the surface also varies at different planes for a particular build orientation, as shown in Fig. 8(f) and (g). This indicates that researchers and engineers must adopt a specific build strategy.

More widely used 3D printing techniques for the fabrication of carbon-based polymer composites have been discussed in the previous sections. However, there are some other techniques also which can also be used. 
(a)

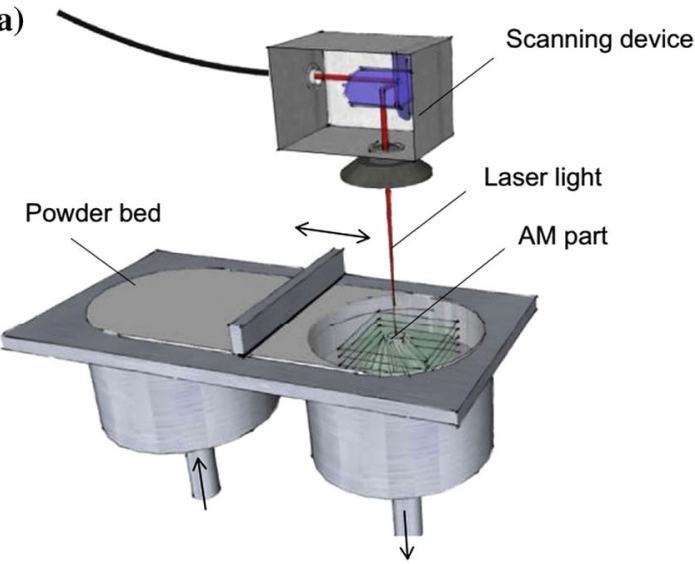

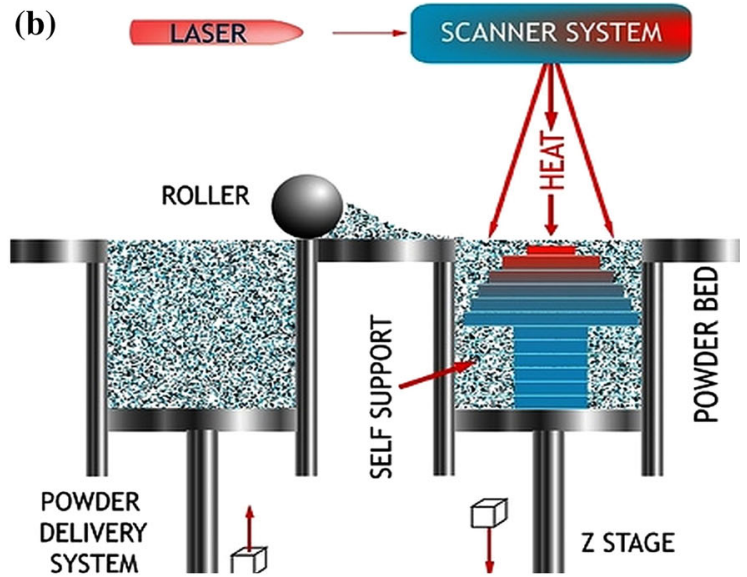

(c)

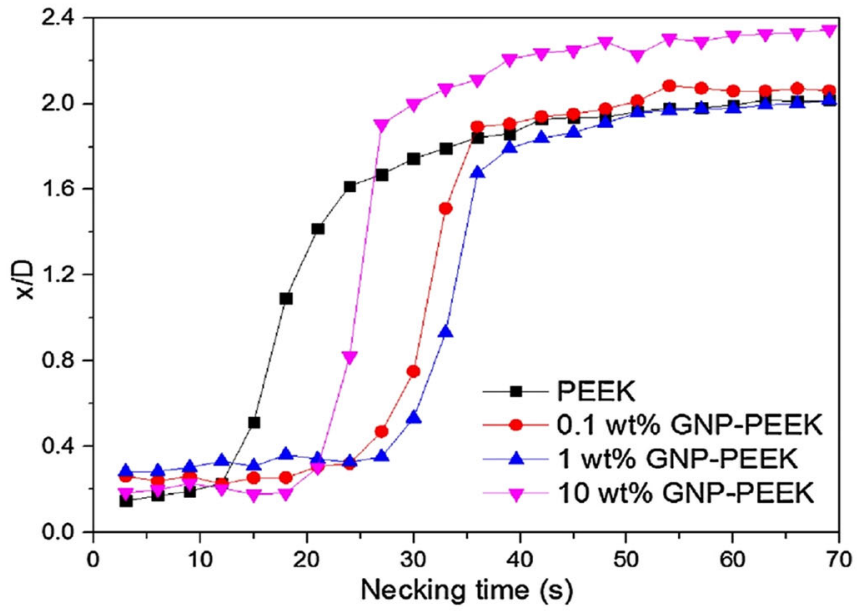

(d)

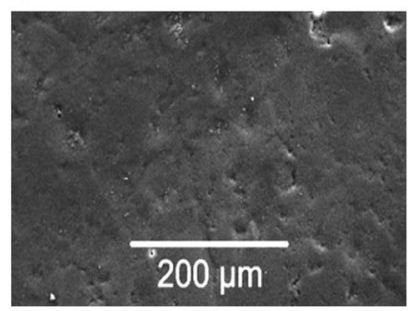

(e)

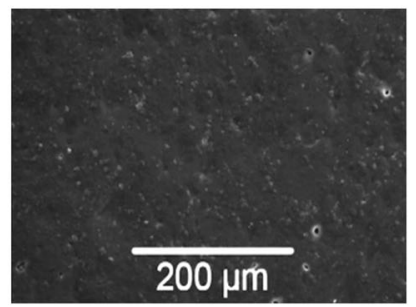

(f)

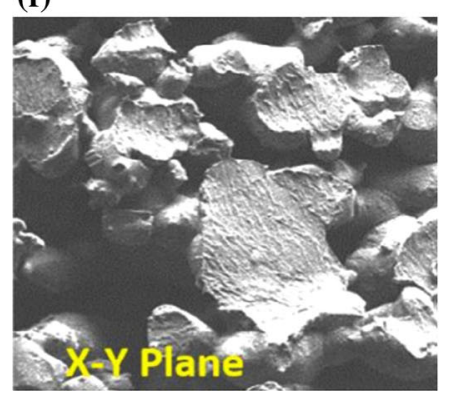

(g)

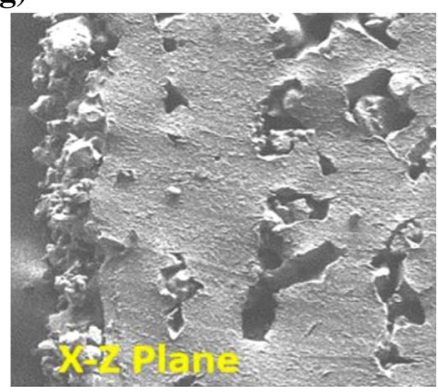

\section{$200 \mu \mathrm{m}$}

Fig. 8 Schematic showing (a) SLS setup and (b) layer building scheme in SLS, (c) curve showing coalescence time results of pure PEEK, 0.1, 1, and $10 \mathrm{wt} . \%$ GNP-reinforced PEEK composites, (d) and (e) show SEM images of pre-PEEK and 1 wt.\% GNP-reinforced PEEK, respectively. (f) and g show SEM images of cross sections of CNTs-reinforced PU composites at X-Y plane and X-Z plane. Panel (a) is reprinted from (Ref 96) with permission from Elsevier, Copyright 2016. Panel (b) is reproduced from (Ref 97). Panels (c), (d), and (e) are reproduced from (Ref 98). Panels (f) and (g) are reprinted from (Ref 93) with permission from Elsevier, Copyright 2017

\section{Carbon-Based Polymer Composites and Their Applications}

Nowadays, most of the research focused on polymer matrix composites is based on carbon-based reinforcement due to their wide range of properties. But it is difficult to build large functional components and complex geometries via conventional methods. The production cost and design-manufacturing cycle are other concerns. To eliminate these limitations, AM methods could be adopted. There are several carbon-based fillers which can be reinforced in polymers and manufactured through the additive manufacturing routes, which are now discussed.

\subsection{Graphene-Based Polymer Composites}

Graphene is considered as fascinating material in many technical fields. It is a two-dimensional monolayer of $\mathrm{sp}^{2}$ hybridized carbon atoms which are arranged in a honeycomb 

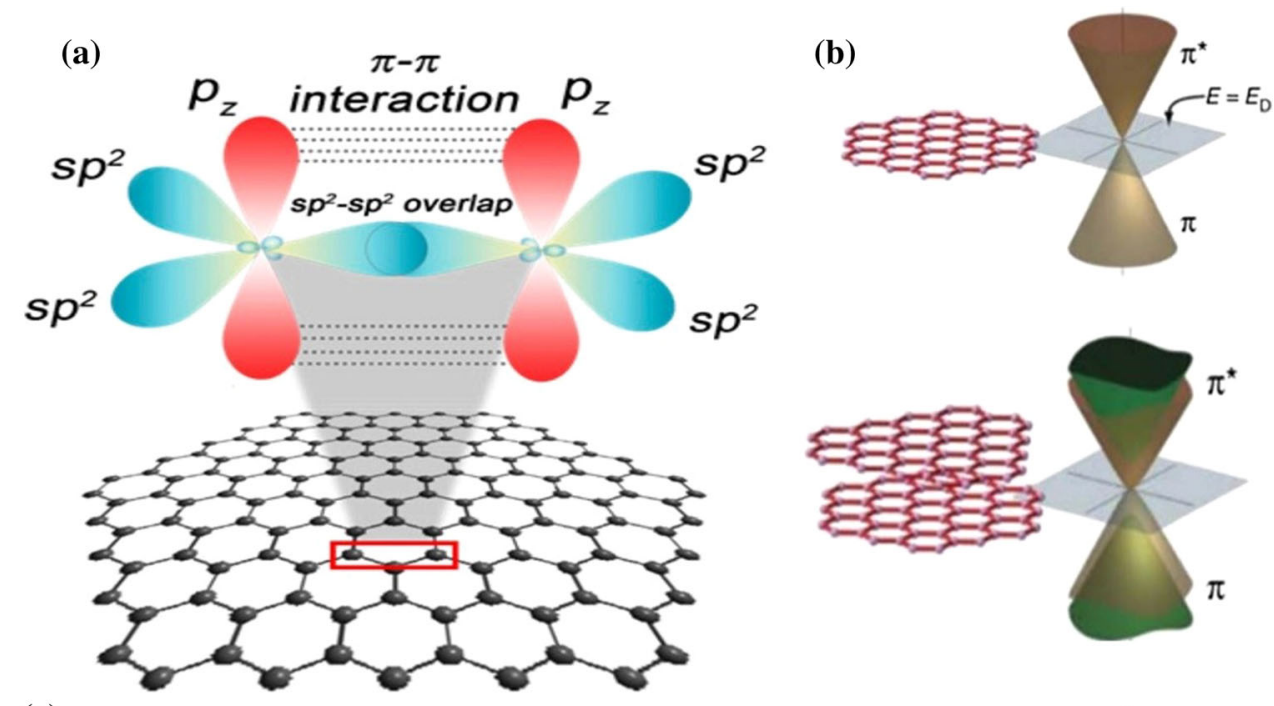

(1)
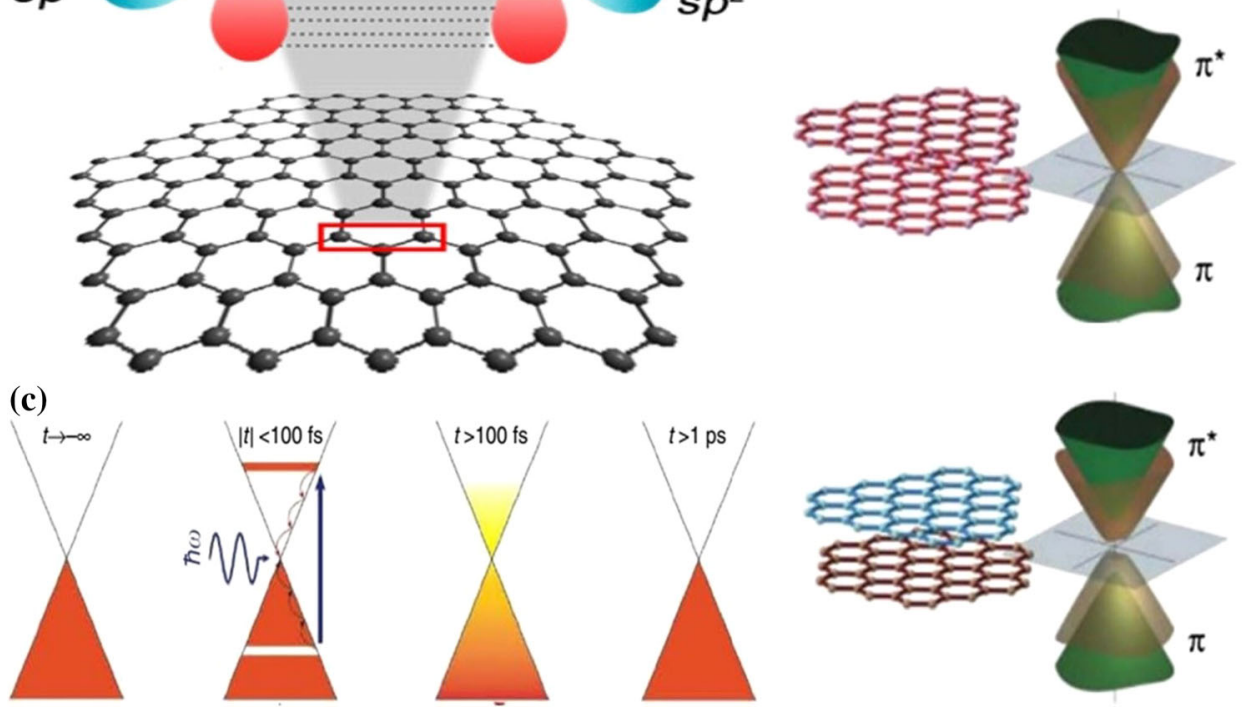

Fig. 9 Schematic showing (a) pristine graphene with $\mathrm{sp}^{2}$-hybridized carbon atoms, (b) electronic structure of graphene and its equivalent and inequivalent AB Bernal stacked layers, and (c) ultrafast temporal dynamics of photoexcited electrons in graphene. Panel (a) is reproduced from (Ref 106). Panel (b) is republished with permission of Royal Society of Chemistry, from "An electronic structure perspective of graphene interfaces,” B.J. Schultz, R.V. Dennis, V. Lee, S.J.N. Banerjee, Vol 17 (10), Copyright 2014 (Ref 107); permission conveyed through Copyright Clearance Center, Inc. Panel (c) is reproduced from (Ref 108)

lattice. It has a typical thickness of $3.4 \mathrm{~A}^{\mathrm{o}}$ with carbon-carbon distance of $1.4 \mathrm{~A}^{\mathrm{o}}$ as shown in Fig. 2 (Ref 99). Graphene is a single and free-standing atomic plane of graphite, see Fig. 9(a), which is isolated from its environment (Ref 100). The electronic structure and its ultrafast temporal dynamics of the photoexcited electron are shown in Fig. 9(b) and (c), respectively. Graphene exhibits supreme properties such as high elastic stiffness $\left(\sim 340 \mathrm{~N} \mathrm{~m}^{-1}\right)$, ultimate tensile strength $(\sim 130 \quad \mathrm{GPa})$, very high thermal conductivity $\left(\sim 5300 \mathrm{~W} \mathrm{~m}^{-1} \mathrm{~K}^{-1}\right)$ and charge carrier mobility $\left(200,000 \mathrm{~cm}^{2} \mathrm{~V}^{-1} \mathrm{~s}^{-1}\right)$ (Ref 101). Thus graphene can be used in a variety of applications ranging from biomedical (Ref 102), electronics (Ref 101), structural (Ref 103), optics (Ref 104), and energy storage devices (Ref 105).

Although graphene has attractive properties, its restacking issue has limited its usage/performance. Thus, to engineer its performance in a variety of applications, graphene is generally formed as a composite with a polymer matrix. The advantages of additive manufacturing over conventional molding techniques for polymer composites, which were discussed in section 1 , have attracted the interest of researchers to fabricate graphene/polymer composites by additive manufacturing.

Wei et al. (Ref 58) fabricated graphene/ABS composite by FDM. An electrical conductivity of $1.05 \times 10^{-3} \mathrm{~S} \mathrm{~m}^{-1}$ was observed for a graphene loading of $5.6 \mathrm{wt} . \%$., where these composites surpassed the percolation threshold (the point at which the filler makes intimate contact). A fall-off in electrical conductivity for less than $2 \mathrm{wt} . \%$ of graphene, as shown in Fig. 10(a), of 3D-printed graphene/ABS composites, was observed due to internal voids being formed between adjacently stacked filaments. Therefore, 3D-printed graphene-ABS composites can be used in applications such as printable electronics and the building of smart structures inside ABS models. SEM images at 3.8 wt.\% loading of graphene revealed partially incorporated dangling graphene sheets.

Energy storage devices can be fabricated by AM, for example, a lithium-ion battery in which electrodes were made of graphene oxide and the electrolyte was a polymer gel fabricated by Fu et al. (Ref 109) using the DIW method. The GO ink was forced to extrude through the nozzle, and a rheological behavior study of GO ink and polymer gel electrolyte showed that they behaved as non-Newtonian fluids with high yield stress and plateau moduli and thus can be printed. Due to high shear stresses induced at the nozzle end, GO flakes were aligned along extruding direction and this directional printing led to an increase in electrical conductivity of the electrode. The printed GO electrode contained pores due to the intrinsically porous structure of the GO flakes, and these pores acted as housing sites for electrolyte (polymer gel). As full cells with the different number of layers (same layer thickness) were fabricated, the total thickness of the cell exhibited a linear relationship with the number of layers. This demonstrated the good viscoelastic behavior of the GO and electrolyte inks, which is a prerequisite for $3 \mathrm{D}$ printing. The cathode and anode exhibited stable cycling performance with specific capacities of 160 and $170 \mathrm{mAh} \mathrm{g}^{-1}$, respectively. The electrochemical performance of the full cell arrangement is shown in Fig. 10(c) and (d). The initial charge and discharge 

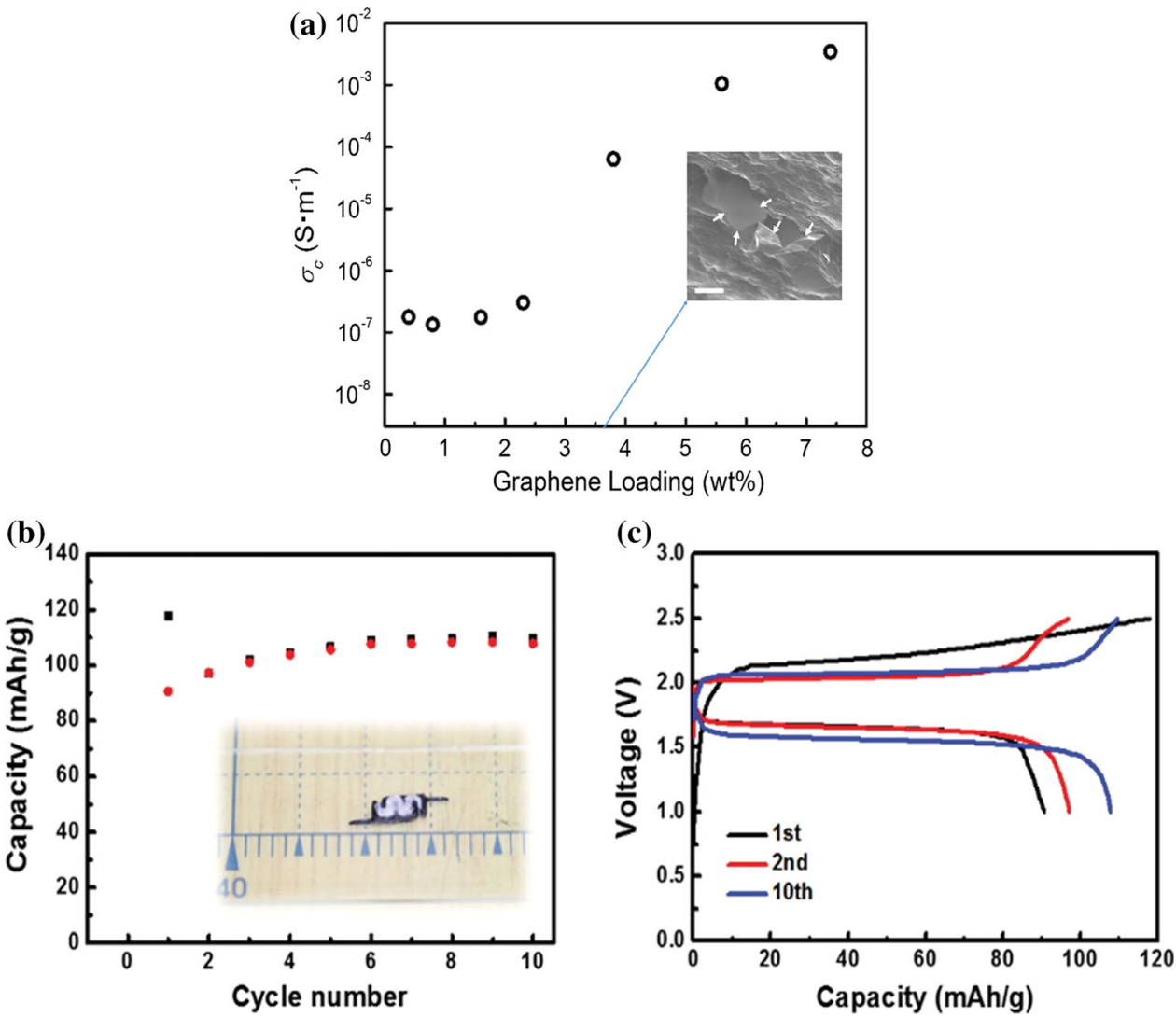

Fig. 10 (a) Electrical conductivity of G-ABS composites as a function of graphene loading, (b) cycling stability, and (c) charge and discharge profiles of the 3D-printed full cell of graphene/polymer electrolyte. Panel (a) is reproduced from (Ref 58). Panels (b) and (c) are reproduced with permission from Ref. (Ref 109). Copyright 2016, WILEY-VCH Verlag GmbH \& Co. KGaA, Weinheim

capacities of 117 and $91 \mathrm{mAh}^{-1}$, respectively, were delivered by a full cell arrangement at a specific current of $50 \mathrm{~mA} \mathrm{~g}^{-1}$. The increase in capacity with number of cycles reflected the enhanced activation of the electrode after a few cycles. Like graphene, other carbon-related materials such as carbon nanotubes and carbon black can also be 3D printed for energy storage applications (Ref 43).

A graphene/epoxy composite fabricated by Compton et al. (Ref 87) using DIW revealed that the rheological behavior of graphene/epoxy composites changed with the percentage of graphene. An increase in graphene content led to an increase in apparent viscosity and also shear thinning. The increase in shear thinning behavior eradicated the need for an excess driving pressure to flow the material from fine scale nozzles.

Graphene-based polymer composites also find their usage in biomedical applications such as drug delivery, gene delivery, cancer therapy, and biosensors (Ref 110, 111). For tissue engineering applications, scaffolds should provide a physical connection for cell infiltration and proliferation. It is difficult to control the number of pores, size and shape of pores, and the distribution of pores in a scaffold using conventional fabrication techniques (Ref 54). The use of 3D printing to create such scaffolds can address these problems and hence can provide structures with better bioactivity as compared to scaffolds fabricated through conventional fabrication techniques.

As an example, a biocompatible graphene scaffold was prepared by Jakus et al. (Ref 111) using DIW printing. The scaffold was comprised of graphene and polylactide-co-glycolide (PLG), and they showed that the graphene/PLG composite can be printed to an overall thickness of $300 \mu \mathrm{m}$. Graphene with a $2 \mathrm{D}$ structure and formed via a uniaxial printing process led to an anisotropic microstructure and anisotropic properties of fabricated composites. The shear forces generated during extrusion led to the alignment of fibers along the surface, whereas there is more random orientation of fibers in conventional cast composites. There was a smooth transition between adjacent layers in terms of physical interaction, which is generally a challenge in 3D printing. Graphene/PLG composite is a biocompatible material and exhibited an intrinsic elastic modulus of $3 \mathrm{MPa}$ and electrical conductivity of $875 \mathrm{~S} / \mathrm{m}$. The conductivity along fiber direction showed an inverse relation with diameter of the extrusion tip. In vitro biocompatibility and bioactivity studies were carried out on graphene/PLG and PLG scaffolds which showed that female bone-marrow-derived human mesenchymal stem cells (hMSCs) remain viable on the graphene scaffolds. The proliferation and viability were significantly higher for graphene/PLG scaffolds as compared to PLG scaffolds. as shown in Fig. 11(a). The surface of 20 vol.\% graphene/PLG scaffold exhibited a confluent sheet-like morphology, see Fig. 11(b), whereas the surface with 60 vol.\% exhibited high aspect ratio cellular extensions on day seven, see Fig. 11(c) and (d). A co-dispersed and surface-modified fillerreinforced polymer scaffolds could also be fabricated via AM techniques. The basic idea of co-dispersing or surface modification of filler is to reduce the aggregation tendency of individual fillers (Ref 112, 113). Shuai et al. (Ref 114) fabricated a co-dispersed (graphene oxide and Ag) poly-Llactic acid (PLLA)-polyglycolic acid (PGA) nanocomposite by 


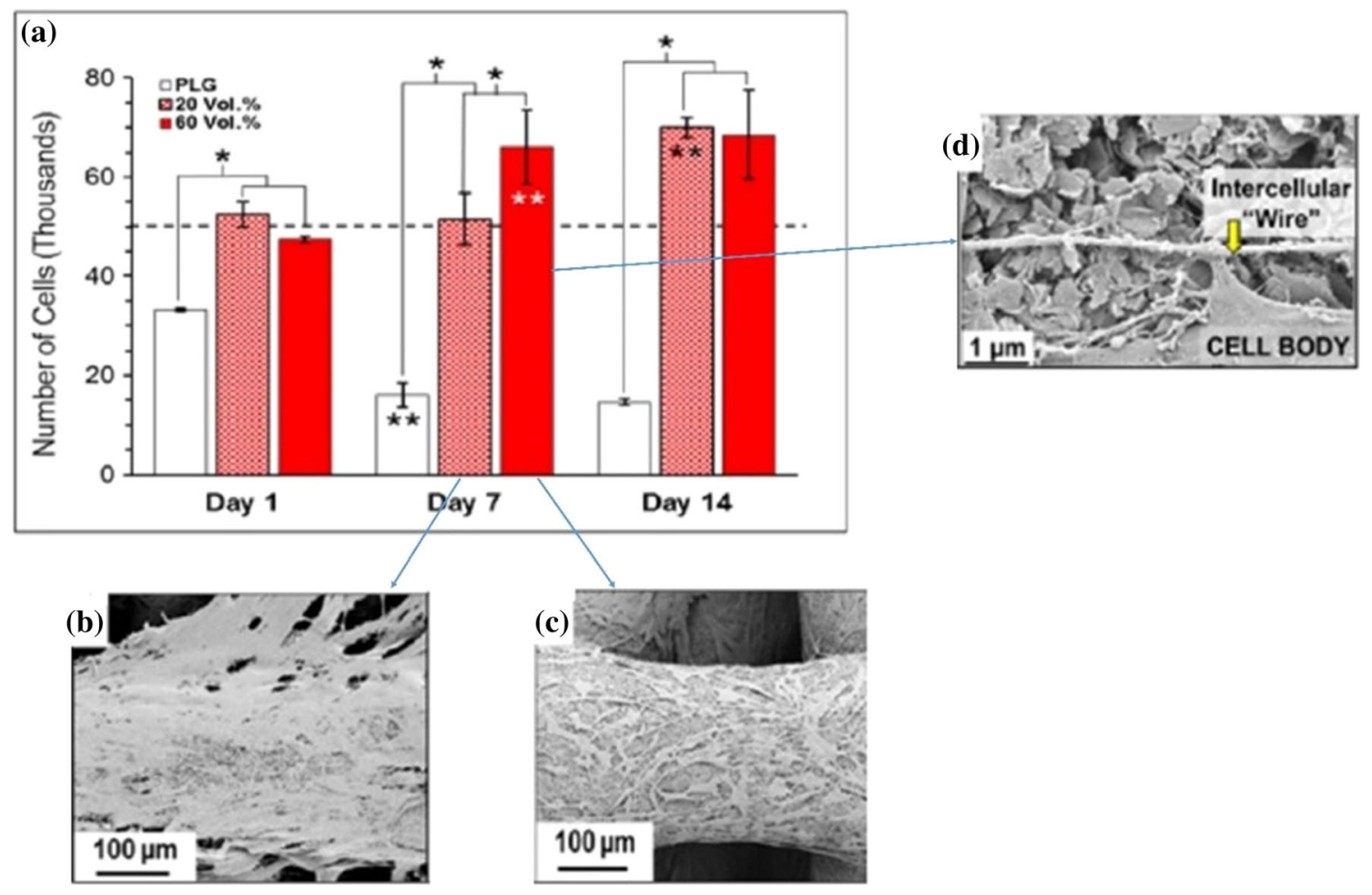

Fig. 11 (a) Number of hMSCs present on graphene/scaffolds $(n=3)$ as a function of material and days after seeding. Dotted line represents initial cell seeding number (50 000), (b) 20, and (c) 60 vol.\% graphene/PLG scaffolds after 7 days, and (d) SEM image of cell on day 7. Reprinted with permission from A.E. Jakus, E.B. Secor, A.L. Rutz, S.W. Jordan, M.C. Hersam, R.N. Shah, Three-dimensional printing of highcontent graphene scaffolds for electronic and biomedical applications, ACS nano, 9(4), 4636-4648 (2015). Copyright 2015 American Chemical Society

SLS. The Ag nanoparticles intercalated into GO interlayers, and GO nanosheets loaded Ag nanoparticles, which increased the interlamellar space of GO nanosheets and interparticle distance among Ag nanoparticles. Hence, aggregation of nanoparticles was significantly reduced and uniform distribution of nanoparticles was achieved. The PLLA-PGA matrix composite with a uniform distribution of reinforcement was realized by SLS. It was observed that co-dispersed PLLA-PGA scaffolds exhibited better performance as compared to $\mathrm{GO}$ and $\mathrm{Ag}$ as individual reinforcements, in terms of compressive strength, antibacterial properties, and cytocompatibility due to homogenous distribution of reinforcement.

Polymer composites reinforced with graphene also exhibit excellent mechanical properties and are lightweight. Thus, graphene/polymer composites are utilized in several mechanical, civil, and aerospace applications (Ref 115). The realization of complex geometries of mechanical structures, especially in aerospace applications, is difficult by conventional fabrication techniques. In addition, these techniques are time-consuming and costly. To address these problems, 3D printing has been used to fabricate intricate structures.

Dul et al. (Ref 24) fabricated graphene/ABS composite by FDM. They showed that the mechanical properties of graphene/ ABS composites change with the orientation of layers and how the layers were built up. Composites which were horizontally built exhibited the highest elastic modulus and ultimate tensile strength followed by vertical and then perpendicular parts, respectively. The elastic modulus of graphene/ABS composite was increased by 32,28 , and $8 \%$ for horizontal, vertical, and perpendicularly built-up parts, respectively, as compared to pure ABS. The ultimate tensile strength values also followed the same pattern because fracture behavior and strength values depend upon the load at which the layers debond which in turn depends upon the orientation of layers.

A graphene oxide/photopolymer composite was fabricated by Lin et al. (Ref 75) using a mask projection-based stereolithography system. Prior to tensile sample fabrication, they printed a truss using a neat polymer and a graphene oxidereinforced polymer, as shown in Fig. 12(a) and (b), respectively, to examine the variability of the mask projection-based stereolithography system. The tensile strength of $0.5 \mathrm{wt} . \%$ graphene oxide-reinforced polymer composite was $12.99 \mathrm{MPa}$, which was $45.3 \%$ higher as compared to neat polymer, see Fig. 12(c). The tensile strength further increased when postheat treatment of the composite was undertaken which increased interfacial strength between different layers. The microstructure of the composite revealed that graphene oxide was randomly distributed in the matrix and was not fully flat, i.e., it was wrinkled. TEM images of graphene oxide/polymer composite are shown at the edge (Fig. 12d) and middle (Fig. 12e) of the TEM sample, which indicated the wrinkling of graphene oxide in printed composites.

Polyamide-12/functionalized graphene nanoplatelet composite fabricated by Kim et al. (Ref 116) using the SLS technique showed that a large amount of reinforcement particles may lead to defect formation, such as agglomeration and irregular melting, in the composite during fabrication. Irregular melting can affect the rheological and thermal behavior of the base material. The stress-strain behavior of fabricated composite is shown in Fig. 12(f). Reinforcing 


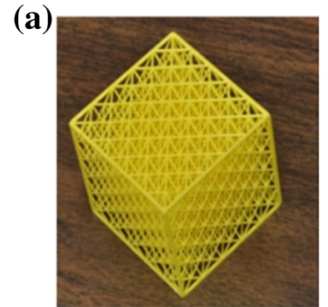

(b)

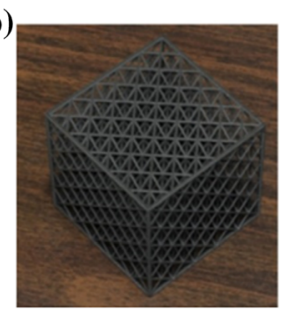

(c)

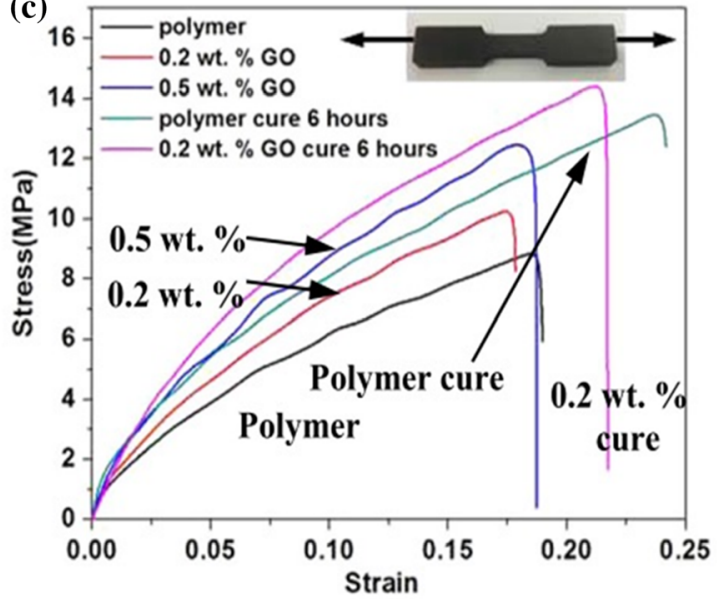

(d)

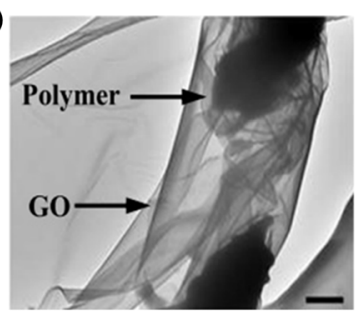

(e)

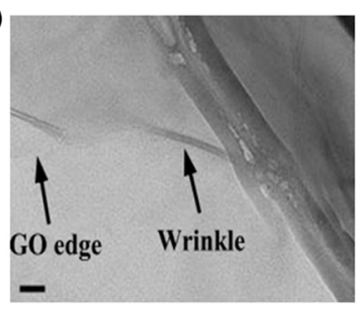

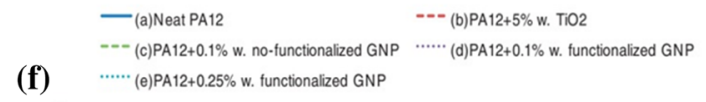

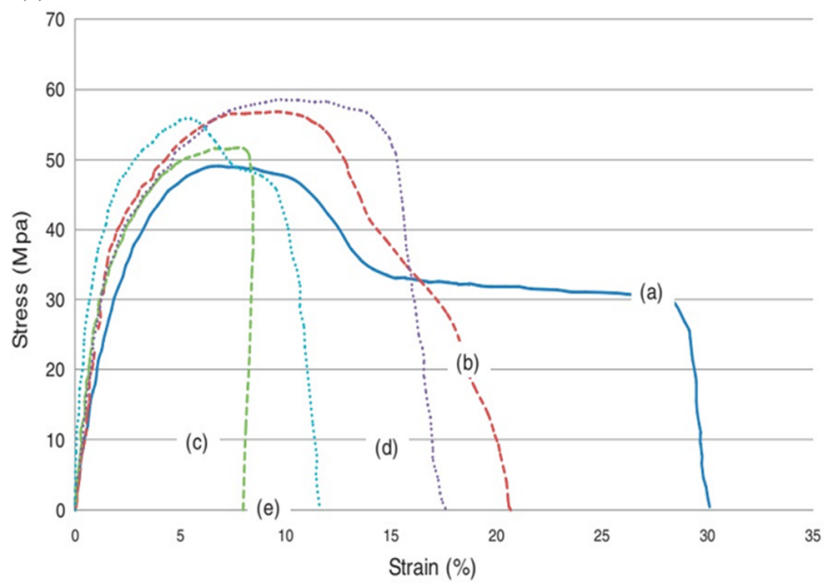

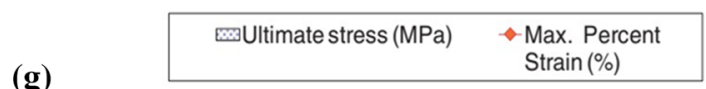

(g)

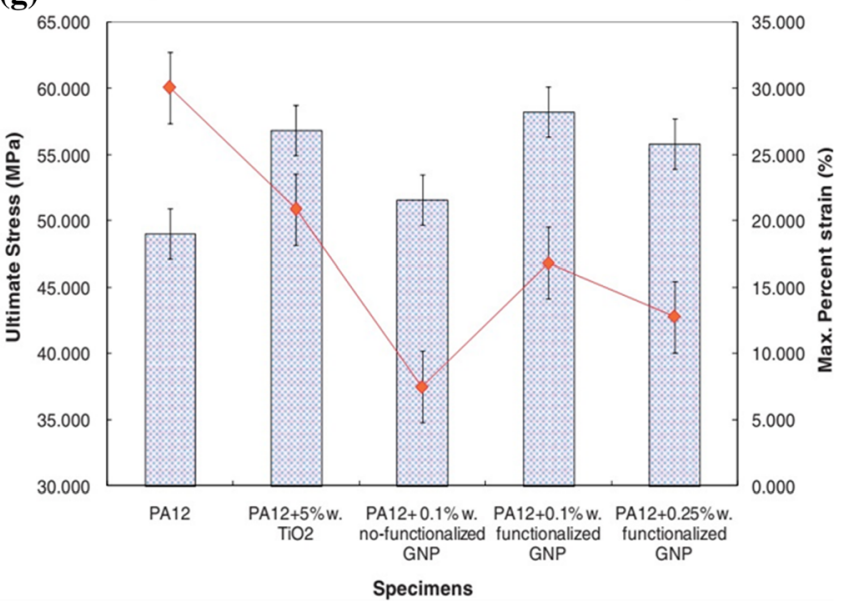

Fig. 12 Panel A; (a-e): (a) polymer and (b) GO-reinforced polymer trusses fabricated by mask projection-based stereolithography system, (c) tensile stress-strain behavior of polymer and corresponding GO-reinforced polymers, before and after post-curing, (d) stress-strain behavior, and (e) ultimate tensile strength of GNP/PA-12 composites fabricated by SLS technique. Panel B; (f, g): (f) stress-strain behavior of PA12/functionalized composites, and (g) its ultimate strength. Panel A is reprinted from (Ref 75). (C) IOP Publishing. Reproduced with permission. All rights reserved. Panel B is reproduced with permission from (Ref 116)

$0.1 \mathrm{wt} \%$ of functionalized graphene increased the ultimate strength by $15.8 \%$ as compared to neat PA-12, as shown in Fig. 12(g).

\subsection{Carbon Nanotubes-Based Polymer Composites}

Carbon nanotubes (CNTs) are seamless tubes comprised of one or more layers of graphene (designated as single-wall, SWNT, or multiwall, MWNT) with closed or open ends (Ref 117). Conceptually, carbon nanotubes can be infinitely long; thus, it can be represented as a one-dimensional system as shown in Fig. 2 (Ref 118). All carbon atoms are bonded in a hexagonal lattice in a perfect CNT, except at the ends. However, mass-produced CNTs also contain certain defects such as pentagons, heptagons, and other imperfections in the sidewalls which can degrade the properties of CNTs. The length of CNTs can vary from less than $100 \mathrm{~nm}$ to several centimeters with diameter ranging from 0.8 to $2 \mathrm{~nm}$ (SWNTs) and 5 to $20 \mathrm{~nm}$ (MWNTs). Thus, CNTs can have an aspect ratio greater than 1000 (Ref 118). At least one end of the cylindrical tube is capped with a hemisphere having fullerene structure.
CNTs can have metallic or semiconducting nature depending upon its chirality, i.e., orientation of the graphene lattice with respect to the tube axis. It can have three chiralities, i.e., zigzag, armchair, and chiral one, as shown in Fig. 13(a). Although CNT is made of graphene lattice, its band structure is different to graphene, as shown in Fig. 13(b).

CNTs are known for their high mechanical properties. Its elastic modulus approaches $1 \mathrm{TPa}$, and its tensile strength is nearly $100 \mathrm{GPa}$ (Ref 119). This strength is about 10 times more than that of any industrial grade fiber. Apart from its excellent mechanical properties, CNTs also exhibit excellent physical properties. The room temperature thermal conductivity of SWNT can be $3500 \mathrm{~W} \mathrm{~m}^{-1} \mathrm{~K}^{-1}$ (Ref 120), and CNTs also exhibits excellent electrical conductivity and almost negligible thermal expansion coefficient (Ref 118). These outstanding properties of CNTs make it a potential candidate for a wide variety of applications. However, there are some issues that must be addressed before applying them on the macroscale. Poor alignment and contact resistance between individual CNTs lead to phonon and charge carrier scattering (Ref 121). 


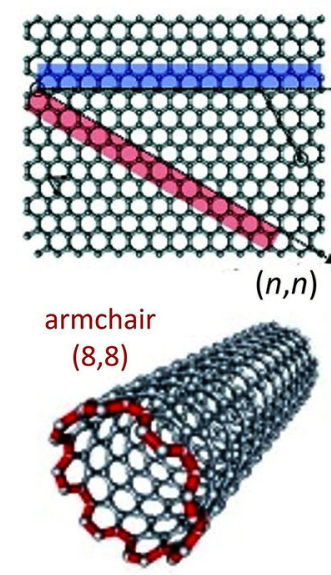

(a)
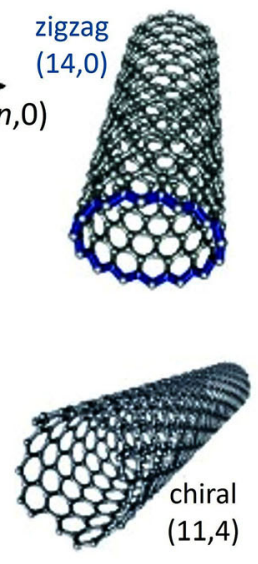

$(11,4)$

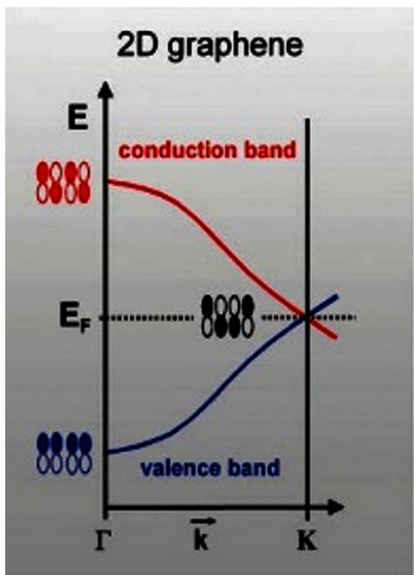

(b)

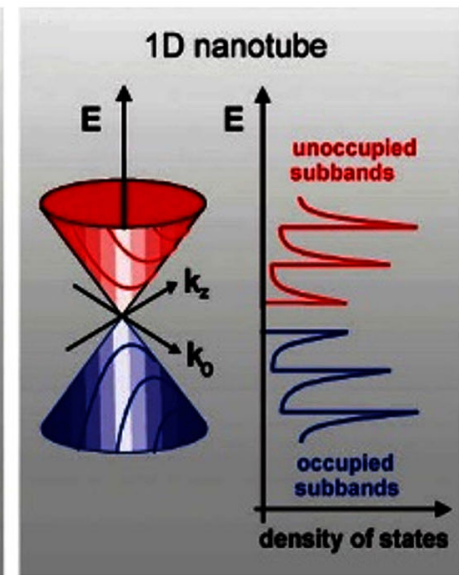

Fig. 13 (a) Rolling up of 2D graphene sheet leads to three different types of CNTs, (b) schematic representation for band structure comparison of 2D graphene sheet and 1D carbon nanotube. Panel (a) is reprinted from (Ref 122) with permission from Elsevier, Copyright 2015. Panel (b) is reproduced with permission from (Ref 123). Copyright 2009, WILEY-VCH Verlag GmbH \& Co. KGaA, Weinheim

(a)
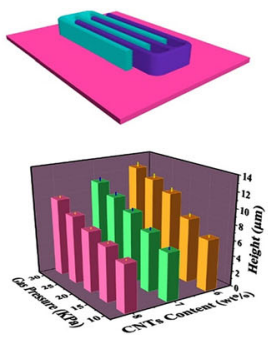

(b)

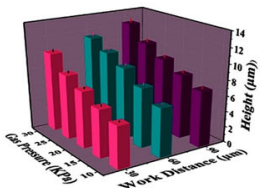

(c)
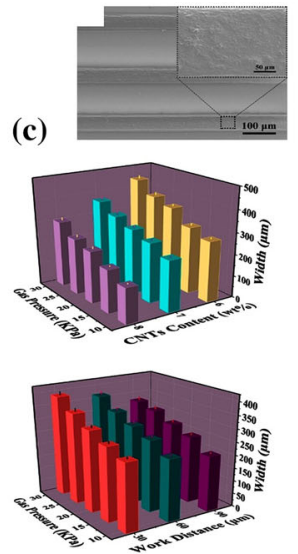

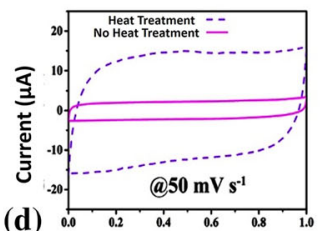

Voltage (V)

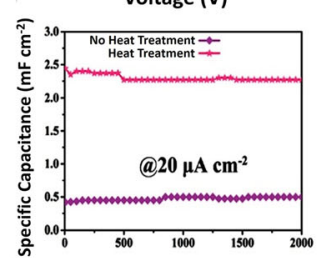

(e)

Cycle Number (f)
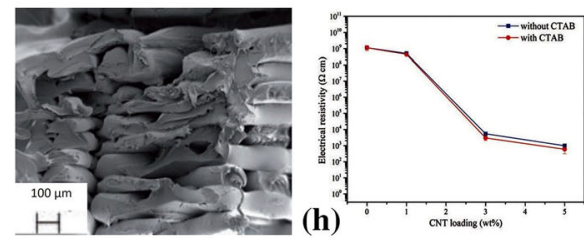

(g)
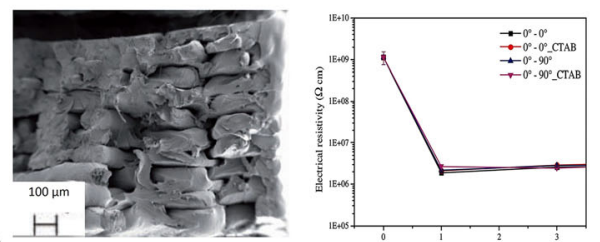

(i) (i)

Fig. 14 Panel A; (a-e): (a) schematic showing as-obtained interdigital electrodes of CNTs/PVA for microsupercapacitors, (b) influence of gas pressure on geometric features of CNTs/PVA microsupercapacitor electrodes, (c) SEM image showing microsupercapacitor electrode, (d) the CV curves of the packed supercapacitors, (e) the cyclic stability performance of the packed supercapacitors. Panel B; (f-i): (f) neat PVA polymer, and (g) 1 wt.\% CNT-reinforced PVA printed by FDM, electrical resistivity of (h) single-printed filament, and (i) 3D-printed composite sample. Reprinted with permission from W. Yu, H. Zhou, B.Q. Li, S. Ding, 3D printing of carbon nanotubes-based microsupercapacitors, ACS applied materials \& interfaces, 9(5), 4597-4604 (2017) (Ref 130). Copyright 2017 American Chemical Society. Panel B is reproduced with permission from (Ref 26)

This results in deterioration of its performance in terms of thermal and electrical conductivity. Further topological defects affect its mechanical properties, and even a small number of them can reduce the mechanical properties significantly. To address these issues, CNTs are generally formed as a composite with other materials.

Particulate reinforced polymer matrix composites are being used in a wide variety of applications (Ref 124), and CNTs are a potential reinforcement in polymer matrix composites for a variety of applications (Ref 125-129). Yu et al. (Ref 130) fabricated CNTs-reinforced polyvinyl alcohol (PVA) composites for microsupercapacitor applications using the DIW technique. A schematic showing the microcapacitor is shown in Fig. 14(a) with its SEM image in Fig. 14(c). The authors found that the geometric features (height and width) of 3Dprinted parts increase with increase in the gas pressure (i.e., nozzle pressure) as can be seen in Fig. 14(b). 8 wt.\% of CNT loading was found to be optimum between viscosity and rheological behavior of ink.

The authors (Ref 130) also showed that post-heat treatment increases the adhesion between filaments and thereby improves the properties of the composite. The electrochemical performance of fabricated microsupercapacitors is shown in Fig. 14(d) and (e). Fabricated composites exhibited an area capacitance of $0.418 \mathrm{mF} \mathrm{cm}$ cm $^{-2}$ at a current density of $20 \mu \mathrm{A}$ $\mathrm{cm}^{-2}$, whereas post-heat-treated composites exhibited an area capacitance of $2.44 \mathrm{mF} \mathrm{cm}^{-2}$ at the same current density, see Fig. 14(e). This increase in area capacitance was attributed to improved contact between the CNTs and electrolyte due to post-heat treatment.

Rigotti et al. (Ref 26) also fabricated CNTs-reinforced polyvinyl composites by FDM. They found that the extruded filaments were more like flattened circles when the neat polymer was extruded, see Fig. 14(f). CNTs increased the 
viscosity of polymer, and this increased the resistance of the material to be extruded through the nozzle. Thus, as the percentage of CNTs was increased, the filaments became more elliptical, see Fig. 14(g). The storage and loss modulus were not affected by the percentage of CNTs, and the electrical resistivity of composite filaments was lower than 3D-printed samples as shown in Fig. 14(h) and (i). The high value of electrical resistivity for 3D-printed samples was attributed to the enriched regions of CNTs in the printed filaments which were shielded by an external layer of polymer.

Currently, CNTs are also used for biomedical applications. Nanophase biomaterials have higher biocompatibility and bioactivity as compared to their micro- and macro-phase counterparts (Ref 131). Researchers have investigated the interaction of CNTs with several mammalian cells and animals and have demonstrated positive results in terms of biocompatibility and bioactivity (Ref 132-134). CNTs can promote cell adhesion and inhibits bone resorption by decreasing osteoclast number (Ref 135-137). Thus, composites using biocompatible polymers find applications in biomedical sectors. 3D printing has some significant advantages over conventional techniques to fabricate $3 \mathrm{D}$ intricate microarchitectures for biostructures, as described in section 3.1.

Yildirim et al. (Ref 33) fabricated a SWNTs-reinforced alginate scaffold using a multi-nozzle biopolymer deposition technique, see Fig. 15(a). They observed that the 3D-printed CNTs/alginate composite exhibited a large surface area and rougher surface as compared to pure alginate. The morphology of SWCNT/alginate scaffold is shown in Fig. 15(b). The cell viability and proliferation data showed that rat heart endothelial cells (RHEC) continuously proliferated at a constant rate up to one week and its number, on day seven, was increased by more than six times as compared to its number at day zero, as shown in Fig. 15(c). The cell proliferation rate of SWCNTs/alginate was higher than that of pure alginate since the SWCNTs increased the material defects and electron delocalization at the surface. The AM techniques could also be used to construct nanosandwich structures with two types of reinforcements. These nanosandwich structures may result in increased surface area due to negligible aggregation of reinforcement and hence beneficial for applications in scaffolds. In a research work, Feng

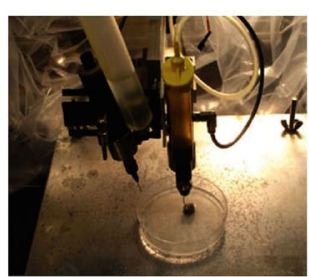

(a)
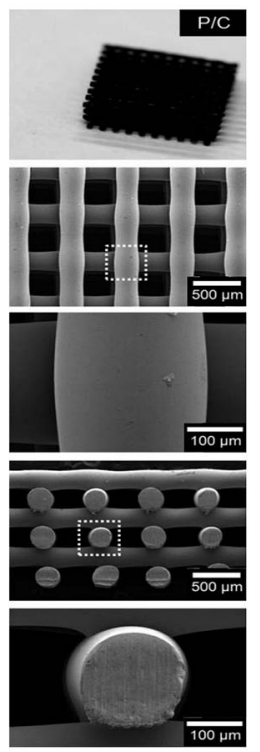

(d)

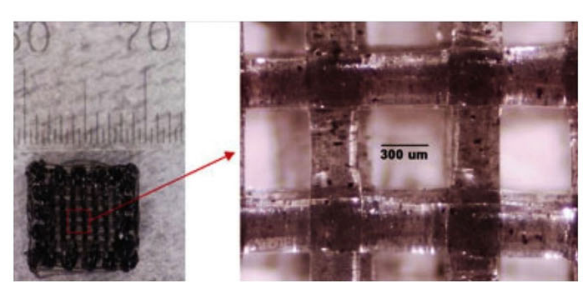

(b)
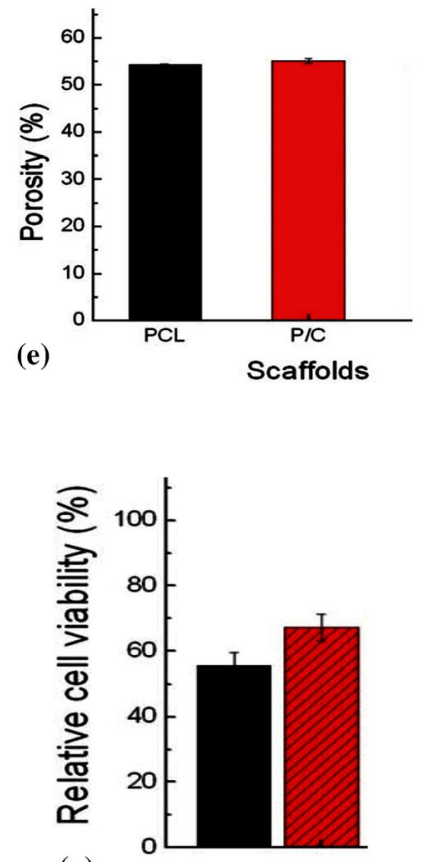

(f)

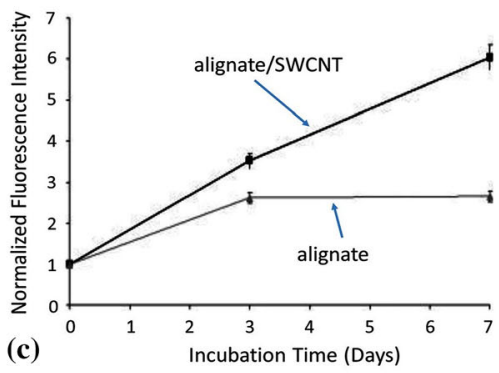

(c)

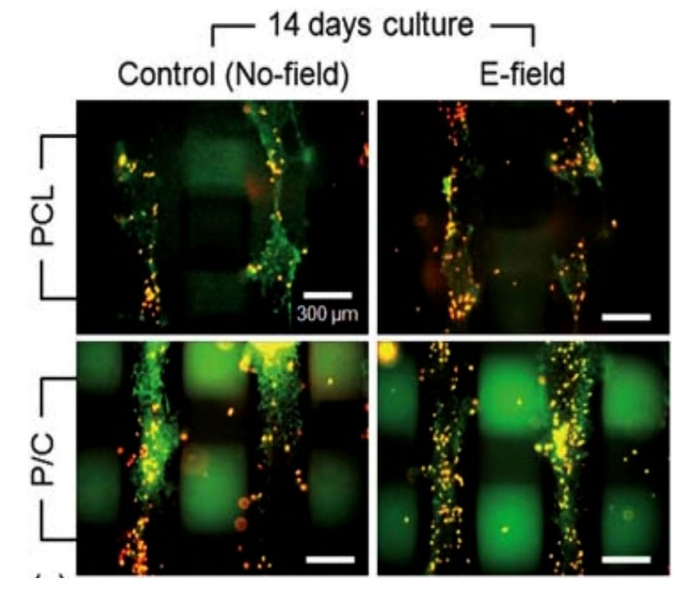

(g)

Fig. 15 Panel A; (a-c): (a) schematic of in situ dual-nozzle cross-linking, (b) morphology, and (c) rat heart endothelial cell (RHEC) viability and proliferation of SWCNTs/alginate scaffold. Panel B; (d-g): (d) optical, surface, and cross-sectional SEM micrographs, (e) porosity, (f) live (green) and dead (red) MG63 cells after 14 days of culture, with and without electric stimulation, and (g) relative cell viability of electrically stimulated scaffolds normalized with control scaffolds (black: PCL, red: P/C) for P/C (PCL/CNT) and PCL scaffolds. Panel A is reproduced with permission from (Ref 33). Copyright 2008 Wiley Periodicals, Inc. Panel B is republished with permission of Royal Society of Chemistry, from The effect of sinusoidal AC electric stimulation of 3D PCL/CNT and PCL/ $\beta$-TCP based bio-composites on cellular activities for bone tissue regeneration, G. Jin, G. Kim, Vol 1 (10), Copyright 2013 (Ref 139); permission conveyed through Copyright Clearance Center, Inc 
et al. (Ref 138) fabricated hydroxyapatite-polyetheretherketone (HAP-PEEK) scaffolds with a nanosandwich structure of 2D graphene nanosheets and 1D CNTs as reinforcements, via SLS. The graphene nanosheets were penetrated by tubular CNTS which resulted in reduced aggregation and increased surface area due to which the cells spread well and attached on the surface of the scaffolds and showed good viability, proliferation, and differentiation.

Jin et al. (Ref 139) fabricated 3D CNT/polycaprolactone (PCL)-based biocomposite by the melt-plotting system and investigated the effect of sinusoidal AC electric stimulation on cellular activities for bone tissue regeneration. Microscopic images of CNT/PCL are shown in Fig. 15(d), and if the percentage of CNT in the mixture was more than $0.2 \mathrm{wt} \%$, then it was difficult to extrude the mixture via nozzles. The measured average pore size $(313 \pm 12 \mu \mathrm{m})$ and porosity $(53 \pm 2 \%)$ were not identical to the designed values (pore size; $300 \mu \mathrm{m}$ and porosity; 55\%) in the CAD system, but were in a reasonable range, and are shown in Fig. 15(e). The highest bone mineralization was observed for stimulated scaffolds as compared to non-stimulated scaffolds. Figure 15(f) shows the dispersion of dead and live cells after 14 days of cell culture. It can be observed that for the case of electric field stimulated scaffolds, dead cells were widely spread and this increased the cell viability, as shown in Fig. 15(g).

CNTs are mostly known for their excellent mechanical properties. However, the realization of free-standing mechanical structures is very difficult. The impressive properties of CNTs make it a material of choice for reinforcement in composite materials (Ref 127), and CNT-reinforced polymer composites have been investigated for their mechanical properties (Ref 125, 140, 141). 3D-printed CNTs/polymer composites have also been studied for their mechanical properties.

Zhang et al. (Ref 142) fabricated CNT/ABS nanocomposites by FDM, and composites fabricated by FDM generally exhibit a weak interlayer adhesion which results in delamination and mechanical failure of the composites. A post-heat treatment of FDM-printed composites (to enhance the bonding between filaments) may resolve this issue, but the probability of deformation increases with bulk heating.

Thus, to address this problem, Zhang et al. (Ref 142) treated 3D-printed CNT/ABS nanocomposite under the application of microwave radiation. A large amount of heat was generated in the vicinity of the carbon nanotubes for a short period of time which melted the ABS matrix adjacent to the CNTs and enhanced the interlayer adhesion. Tensile strength of approximately $34 \mathrm{MPa}$ was observed for a composite having 5.85 wt.\% of CNT treated for $6 \mathrm{~s}$ under microwave radiation, Fig. 16(a), whereas the untreated composite possessed a tensile strength of $\sim 30 \mathrm{MPa}$. The same composite showed an $8.8 \%$ increase in flexural strength after microwave treatment, see Fig. 16(b). These SEM images in Fig. 16(c) for composite with and without microwave irradiation showed an enhancement in interlayer adhesion with microwaves.

Sandoval et al. (Ref 72) used an SLA technique to fabricate MWNTs-reinforced photocross-linkable epoxy resin composites. They observed a variation in the surface finish for different faces of a printed composite. The upper surface exhibited a glossy finish, whereas the lower surface showed a dull or rough surface. The side faces exhibited a layered surface. Part deformation and curling were also observed due to the build-up of internal stresses as the laser cure depth was too great. Attractive forces between graphitic structures of nanotubes due to extended $\pi$ electron systems led the nanotubes to form a fiber-like structure in the composite, and TEM analysis showed good wetting between MWNTs and epoxy. The interface bonding between the highly cross-linked epoxy resin and MWNTs was therefore strong. The observed ultimate tensile strength for MWNTs/epoxy resin composites was 17\% higher than that of pure resin, whereas the breaking strength was $37 \%$ higher, as shown in Fig. 16(d). An effective load transfer via shear mechanisms was observed, and due to the random orientation of nanotubes, it was also observed that composite exhibited almost isotropic properties regardless of its build orientation.

\subsection{Carbon Fiber-Reinforced Polymer Composites}

Among various types of reinforcements in polymers, fibers are considered to be the best choice for polymer matrix composites, especially for mechanical structures (Ref 143). Fiber-reinforced composites exhibit superior mechanical properties as compared to conventional structural materials (Ref 144). Fibers are classified according to their aspect ratio, namely short fibers (discontinuous) or long fibers (continuous). The arrangement of fibers in a matrix may vary according to the application requirement, i.e., random fibers, unidirectional fibers, angle ply laminates, or fibers weaved in different directions. Fiber-reinforced polymer composites have already gained application in aerospace industries, but it can also be used in other applications such as biomedical (Ref 145), electrical (Ref 146), and energy storage devices (Ref 147).

High strength and stiffness, excellent damping properties, low density, and high resistance to corrosion and impact make carbon fiber-reinforced polymer (CFRP) composites a potential candidate for aircraft structures, wind turbine blades, and large civil infrastructures (Ref 146). It also finds applications in various other sectors such as sports equipment, microelectrodes, and automobiles (Ref 148). Carbon fibers are comprised of at least 92 wt.\% of carbon (Ref 149), and their structure may be crystalline, amorphous, or partially crystalline, where the properties depend upon the type of precursor used (PAN, pitchor cellulose-based carbon fiber) (Ref 150). A schematic illustrating the cross section, precursors, and types of carbon fibers is shown in Fig. 17.

The development of various new techniques such as filament winding, bladder-assisted molding, pultrusion, and compression process to fabricate CFRP composites has attracted the attention of many researchers, but these techniques are time-consuming and require extra tooling which makes them an expensive processes; it can also be difficult to make intricate shapes using these techniques, and AM techniques have potential advantages in this regard. Both continuous and short carbon fiber-reinforced polymer composites have been fabricated using AM (Ref 57, 64). Three-dimensional arrangement of carbon fibers can also be realized using AM techniques.

Lewicki et al. (Ref 81) fabricated three-dimensional carbon fiber/polymer composite using a DIW technique with a high degree of fiber alignment. It was observed that $8 \mathrm{wt} . \%$ reinforcement of carbon fiber yields a high resolution in the fabricated part. Composites exhibited highly orthotropic electrical and mechanical responses due to the high degree of fiber alignment. Since carbon fibers possess good electrical conductivity, the electrical conductivity of the polymers can be enhanced by reinforcement with carbon fibers. The electrical 

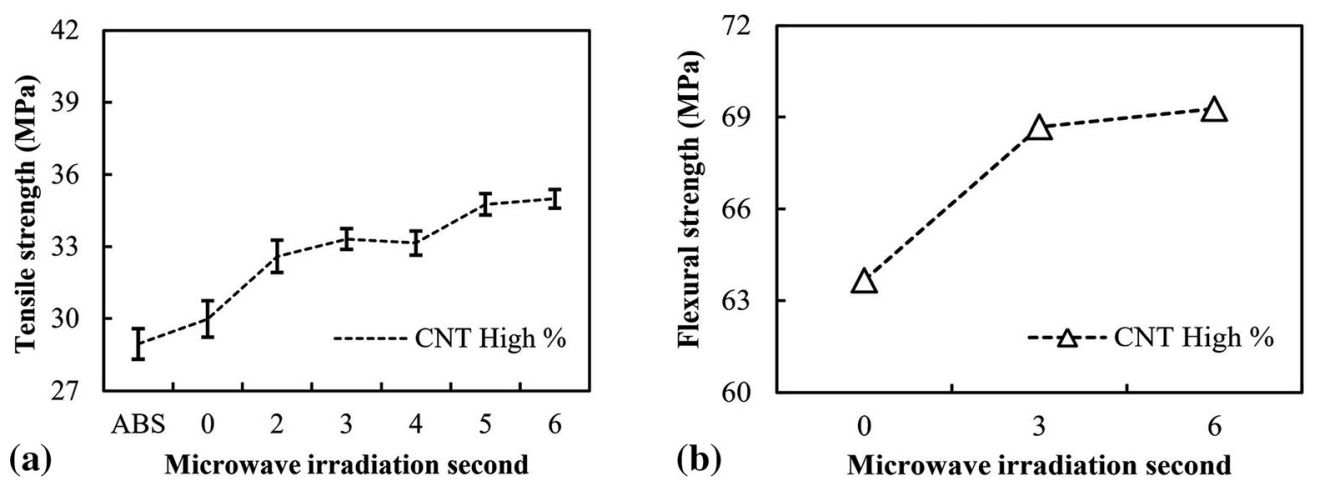

(b)

Microwave irradiation second

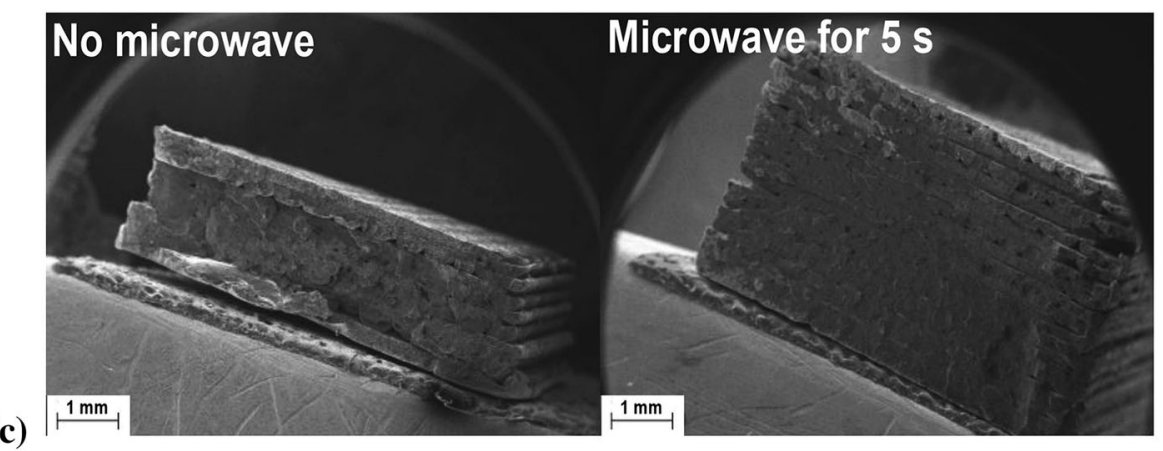

Polymer/MWCNT $(0.05 \mathrm{w} / \mathrm{v})$

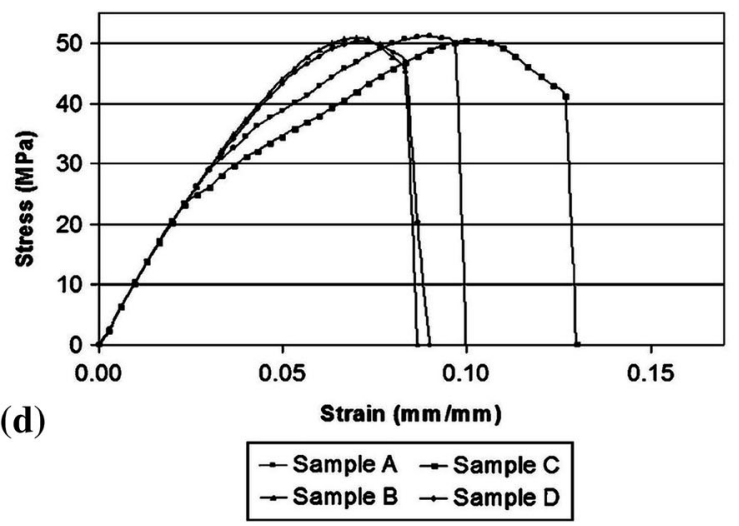

Polymer

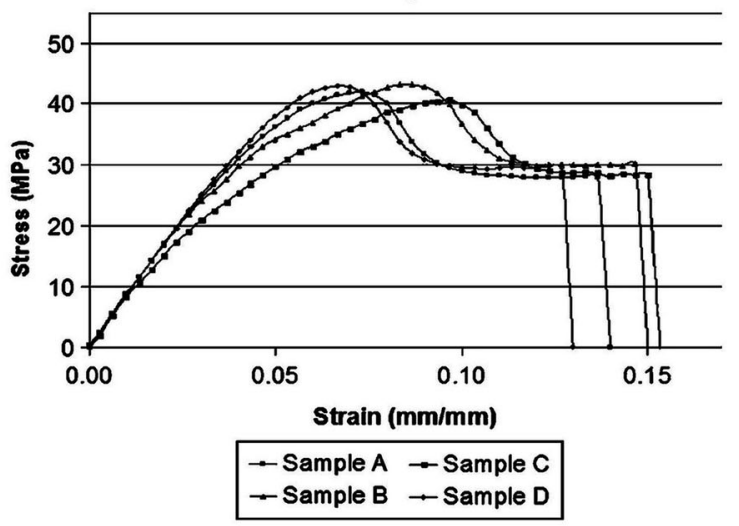

Fig. 16 Panel A; (a-c): effects of microwave irradiation exposure on (a) tensile strength and (b) flexural strength of CNT/ABS nanocomposites, (c) SEM images of CNT/ABS composites for without and with microwave irradiations. Panel B; (d): (d) stress-strain behavior of polymer/ MWCNT and polymer composite under tensile test. Panel A is reprinted by permission from (Ref 142). Copyright 2016, ASME. Panel B is reprinted by permission from Springer Nature: Springer Nature, Journal of Materials Science (Ref 72), Copyright 2006

conductivity of carbon fiber-reinforced polymer composites may vary with the printing direction due to the shear alignment of carbon fibers in the polymer. Carbon fiber/polymer composites fabricated by Lewicki et al. (Ref 81) exhibited average surface conductivity of $1.1 \mathrm{~S} \mathrm{~cm}^{-1}$ in a direction parallel to the printing direction, whereas it was $3.7 \times 10^{-5} \mathrm{~S} \mathrm{~cm}^{-1}$ in the perpendicular direction.

Carbon fiber-reinforced polymers are mainly known for their usage in structural applications due to their high strength and rigidity, and corrosion resistance. An increase in fiber length (in the vicinity of critical fiber length) generally results in an increase in strength of short fiber-reinforced polymer composites, although many other factors are also involved (Ref 154). It is difficult to realize fiber lengths near the critical fiber length for composites fabricated by FDM because high shear mixing of fibers and matrix resin is required prior to feedstock loading. The interaction of fibers with fibers, matrix, and instrument surfaces during shear mixing leads to breakage of fibers. Tekinalp et al. (Ref 64) fabricated carbon fiber-reinforced ABS composite by FDM and compression molding. A schematic showing 3D-printed fiber-reinforced composite by FDM is shown in Fig. 18(a), and the authors used fibers of $3.2 \mathrm{~mm}$ length with $40 \mathrm{wt} . \%$ of fiber loading prior to shear mixing and found that the length of fibers was $\sim 0.4 \mathrm{~mm}$ after shear mixing. The length of the fibers further reduces when the weight percentage of fibers was increased.

Tekinlap et al. (Ref 64) observed that the tensile strength of $3 \mathrm{D}$-printed carbon fiber/ABS composites was $\sim 60 \mathrm{MPa}$ at a 

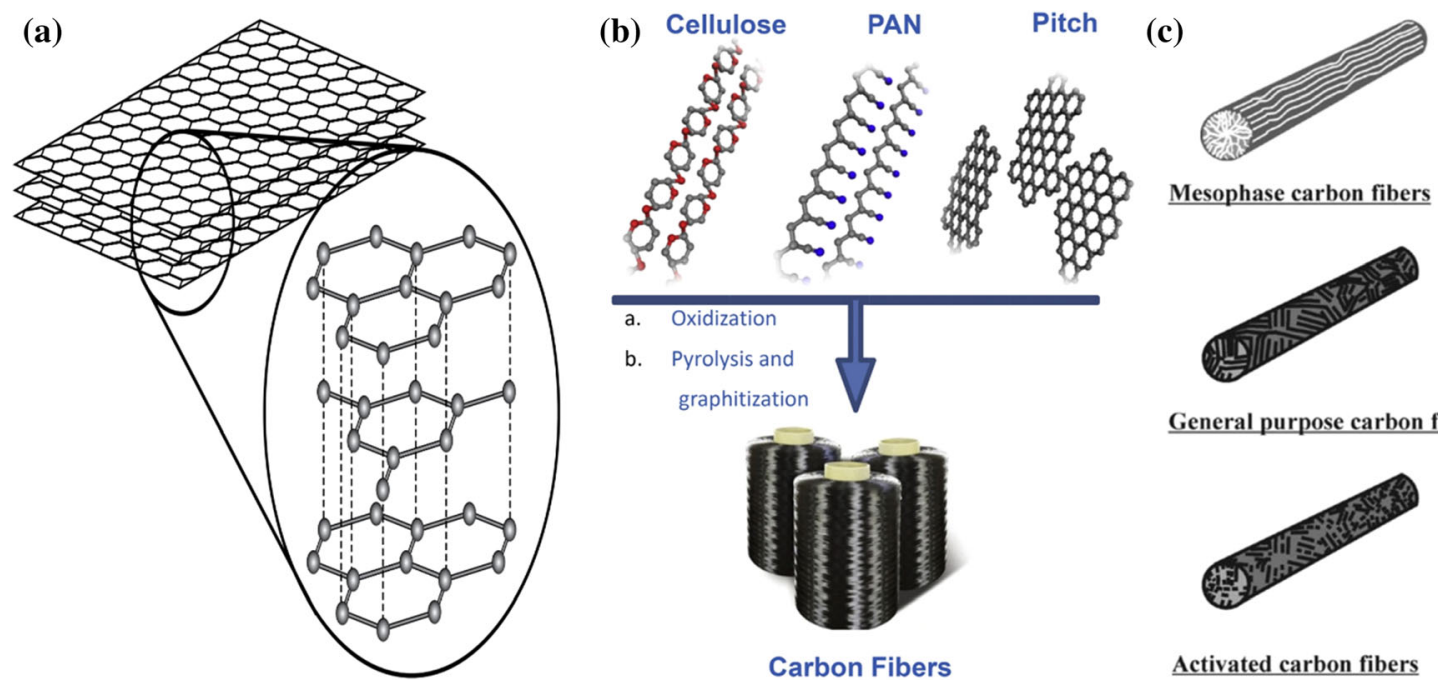

Mesophase carbon fibers

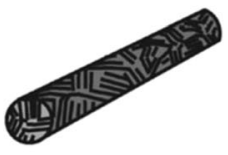

General purpose carbon fibers

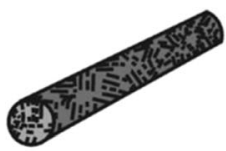

Activated carbon fibers

Fig. 17 (A) Cross section of a carbon fiber with a close-up of the atomic structure, (B) different precursors of carbon fiber, and (C) different types of carbon fibers. Panel A is published with permission ChemMatters (Ref 151). Copyright 2016, ACS. Panel B is reprinted from (Ref 152). Panel C is reprinted from (Ref 153) with permission from Elsevier, Copyright 2001

(a)

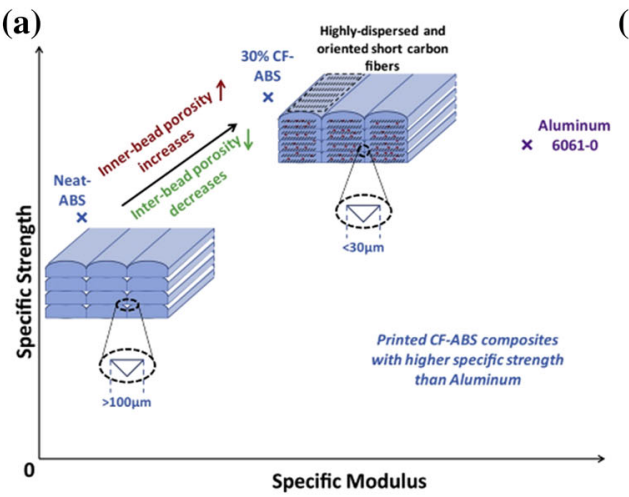

(b)

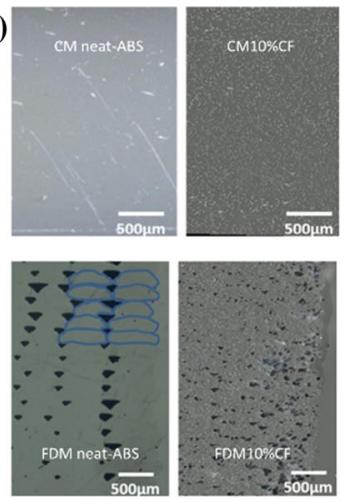

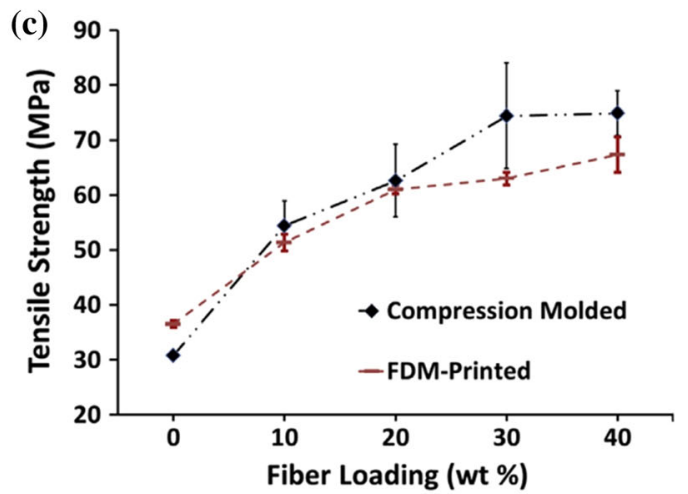

(d)

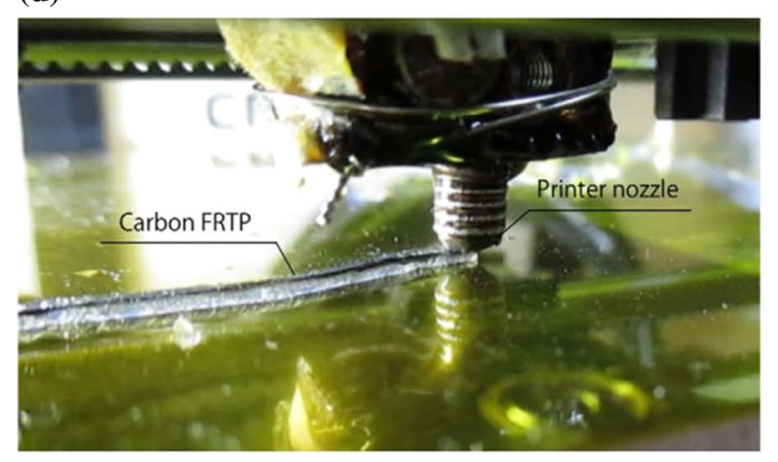

(e)

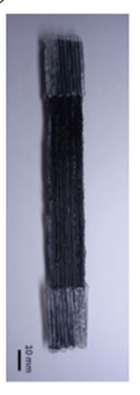

(f)

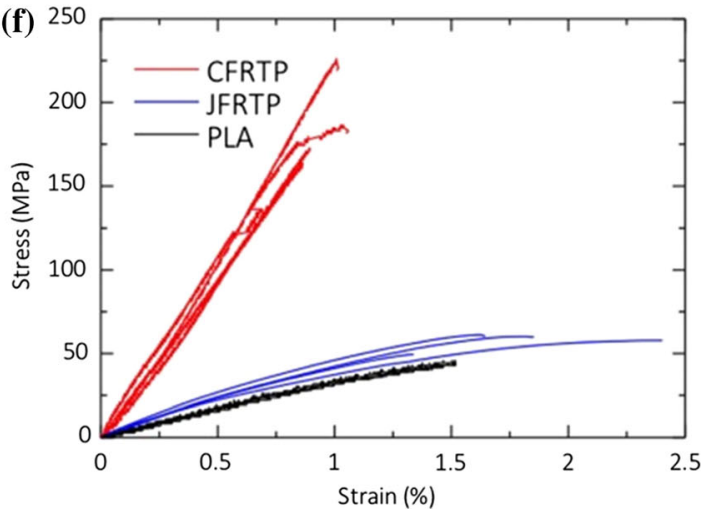

Fig. 18 Panel A; (a-c): (a) schematic presentation of 3D-printed fiber-reinforced composite by FDM, (b) micrographs of polished surfaces of compression molded and 3D-printed carbon fiber-reinforced ABS composites, and (c) their tensile behavior. Panel B; (d-f): (d) photograph of 3D printing of carbon fiber-reinforced thermoplastic polymer (CFRTP) composite, (e) 3D-printed CFRTP, and (c) its tensile behavior. Panel A is reprinted from (Ref 64) with permission from Elsevier, Copyright 2014. Panel B is reproduced from (Ref 4)

fiber loading of $40 \mathrm{wt} \%$, which was lower than compression molded composites, as can be observed from Fig. 18(c). Due to the fiber loading, large voids were observed in FDM printed composites due to resistance to flow at the nozzle side. However, fewer voids were observed in compression molded composites, as can be seen in Fig. 18(b). The relatively high strength of $60 \mathrm{MPa}$ (although it is lower than compression molded composites but higher in the FDM category of short fiber composites) was observed due to highly oriented fibers in composites fabricated by FDM. Besides short fiber-reinforced 
polymers, continuous fiber-reinforced polymers can also be fabricated by AM techniques. Matsuzaki et al. (Ref 4) fabricated continuous carbon fiber-reinforced PLA composites by in-nozzle impregnation. They used a system to supply carbon fibers and resin separately to the printer head as shown in Fig. 18(d).

The printed composites contained $6.6 \%$ of carbon fibers by volume. All fibers were aligned in a single direction, as shown in Fig. 18(e), but the distribution of fibers was not uniform in the entire composite. Further voids were also observed in the printed composites. Tensile tests conducted on printed composites along longitudinal direction showed an increase of 435 and $599 \%$ in tensile strength and tensile modulus, respectively, as compared to neat PLA resin specimens. However tensile strain was decreased to 0.95 (CFRP) from a value 1.45 (PLA), as shown in Fig. 18(f).

Continuous carbon fiber-reinforced nylon composites could also be fabricated by the MarkForged MarkOne system which uses the extrusion technique to print continuous carbon fiber composites with designed architecture. It has been observed by several authors (Ref 155-157) that the composites fabricated by MarkForged system may show a ninefold increase in mechanical properties and relatively less discontinuities as compared to the composites fabricated by FDM. MarkForged system uses two separate printing heads, i.e., one for matrix (nylon or nylon reinforced with chopped carbon fibers), and other for continuous fiber reinforcement. In a research work, Dickson et al. (Ref 157) fabricated continuous carbon fiber-reinforced nylon composites via the MarkForged MarkOne system. They observed a very low level of porosity in the nylon matrix. However, an increase in porosity was observed with an increase in fiber content which may be due to improper impregnation of the matrix while fabricating carbon fiber composite filament. The tensile strength of fabricated composites was significantly higher as compared to the composites fabricated by FDM. Similar results were also observed by Goh et al. (Ref 156) who also observed flattening of filaments after extrusion due to which it is impossible to create thin wall structures by MarkForged MarkOne system.

\section{Summary and Some Critical Issues}

Additive manufacturing has proved itself as most efficient technique for the fabrication of multi-material components/composite materials. High degree of filler alignment in composites can be achieved through AM. These techniques are broadly classified into seven categories, depending on the method of layers build-up.

Carbon-based materials are considered ideal candidates for a variety of applications such as biomedical, structural, electrical, and energy storage applications. The use of carbon-based fillerreinforced polymer composites is increasing at a significant rate whether it is graphene, carbon nanotubes, particulates, or carbon fibers. A variety of AM techniques have been adopted to fabricate carbon-based filler-reinforced polymer composites such as fused deposition modeling, stereolithography, direct ink writing, and selective laser sintering which have been discussed in this review, where each technique has its own advantages and disadvantages and no single technique is an optimum for every composite. Examples of carbon filler-based polymer composites fabricated by AM techniques have been discussed in this review with their performance for specific applications. While AM techniques have attained a significant pace in terms of its development, these techniques are still not widely accepted by most of the industries due to critical issues and challenges which must be resolved. Some of which include:

1. Despite the rapid expansion of AM facilities in the last decade, the range of polymers that can be processed using AM techniques remains relatively limited. Mostly thermoplastic polymers have been processed by AM, and studies on additive manufacturing of thermosetting polymers are limited and these polymers yet to be commercially adopted for AM.

2. Voids and defects are generally present between the interlayers. The adhesion strength between the interlayers can also be improved, where post-curing is often carried out to increase adhesion strength between interlayers.

3. The most commonly used additive manufacturing technique is FDM which has typically low printing resolution (approximately 0.2 to $0.3 \mathrm{~mm}$ ). There are several factors which affect the printing resolution in FDM; these include nozzle diameter, scan speed, and heated chamber temperature. These parameters should be optimized to achieve a high printing resolution. Furthermore, hard carbonaceous fillers can wear out nozzle which affects the printing resolution and must be taken into consideration.

4. Although high degree of fiber alignment can be obtained by additive manufacturing techniques, it remains difficult to obtain fabricated composite with a high degree of particulate filler alignment, which can be necessary to optimize properties and new techniques to tailor filler alignment of composite parts will be beneficial. Furthermore, due to the fabrication nature of AM techniques for fiber-reinforced composites, it is very difficult to achieve a high volume fraction of fibers (i.e., generally $>40 \%$ ) due to which the composites show low properties. Therefore, efficient AM techniques need to be developed which will be able to incorporate high volume fraction of fibers.

5. Due to the difference in the coefficient of thermal expansion between filler and matrix, thermal stresses generate which may lead to deformation of the final product.

The above-stated issues must be resolved for the utilization of AM techniques to its full potential for the fabrication of carbon-based filler-reinforced polymer composites for engineering applications.

\section{References}

1. Z. Quan, A. Wu, M. Keefe, X. Qin, J. Yu, J. Suhr, J.-H. Byun, B.-S. Kim, and T.-W. Chou, Additive Manufacturing of Multi-Directional Preforms for Composites: Opportunities and Challenges, Mater. Today, 2015, 18(9), p 503-512

2. S.E. Zeltmann, K.A. Prakash, M. Doddamani, and N. Gupta, Prediction of Modulus at Various Strain Rates from Dynamic Mechanical Analysis Data for Polymer Matrix Composites, Compos. B Eng., 2017, 120, p 27-34

3. G. Papanicolaou, N. Anifantis, L. Keppas, and T.V. Kosmidou, Stress Analysis of Short Fiber-Reinforced Polymers Incorporating a Hybrid Interphase Region, Compos. Interfaces, 2007, 14(2), p 131-152

4. R. Matsuzaki, M. Ueda, M. Namiki, T.-K. Jeong, H. Asahara, K. Horiguchi, T. Nakamura, A. Todoroki, and Y. Hirano, Three- 
Dimensional Printing of Continuous-Fiber Composites by In-Nozzle Impregnation, Sci. Rep., 2016, 6, p 23058

5. D.W.Y. Wong, H. Zhang, E. Bilotti, and T. Peijs, Interlaminar Toughening of Woven Fabric Carbon/Epoxy Composite Laminates Using Hybrid Aramid/Phenoxy Interleaves, Compos. A Appl. Sci. Manuf., 2017, 101, p 151-159

6. K.C. Warren, R.A. Lopez-Anido, and J. Goering, Experimental Investigation of Three-Dimensional Woven Composites, Compos. A Appl. Sci. Manuf., 2015, 73, p 242-259

7. S. Mortazavian and A. Fatemi, Effects of Fiber Orientation and Anisotropy on Tensile Strength and Elastic Modulus of Short Fiber Reinforced Polymer Composites, Compos. B Eng., 2015, 72, p 116 129

8. E.J. Barbero, Introduction to Composite Materials Design, CRC Press, Boca Raton, 2017

9. S.H. Huang, P. Liu, A. Mokasdar, and L. Hou, Additive Manufacturing and Its Societal Impact: A Literature Review, Int. J. Adv. Manuf. Technol., 2013, 67, p 1-13

10. M. Vaezi, H. Seitz, and S. Yang, A Review on 3D Micro-Additive Manufacturing Technologies, Int. J. Adv. Manuf. Technol., 2013, 67(5-8), p 1721-1754

11. D. Rigotti, A. Dorigato, and A. Pegoretti, 3D Printable Thermoplastic Polyurethane Blends with Thermal Energy Storage/Release Capabilities, Mater. Today Commun., 2018, 15, p 228-235

12. A. Cataldi, D. Rigotti, V.D.H. Nguyen, and A. Pegoretti, Polyvinyl Alcohol Reinforced with Crystalline Nanocellulose for 3D Printing Application, Mater. Today Commun., 2018, 15, p 236-244

13. F. Valentini, A. Dorigato, D. Rigotti, and A. Pegoretti, Polyhydroxyalkanoates/Fibrillated Nanocellulose Composites for Additive Manufacturing, J. Polym. Environ., 2019, 27(6), p 1333-1341

14. V.K. Srivastava, A Review on Advances in Rapid Prototype 3D Printing of Multi-Functional Applications, Sci. Technol, 2017, 7, p 4 24

15. I. Gibson, D.W. Rosen, and B. Stucker, Additive Manufacturing Technologies, Springer, Berlin, 2010

16. F. Ning, W. Cong, J. Qiu, J. Wei, and S. Wang, Additive Manufacturing of Carbon Fiber Reinforced Thermoplastic Composites Using Fused Deposition Modeling, Compos. B Eng., 2015, 80, p 369-378

17. J.Y. Lee, J. An, and C.K. Chua, Fundamentals and Applications of 3D Printing for Novel Materials, Appl. Mater. Today, 2017, 7, p 120-133

18. A.P. West, S.P. Sambu, and D.W. Rosen, A Process Planning Method for Improving Build Performance in Stereolithography, Comput. Aided Des., 2001, 33(1), p 65-79

19. J.P. Kruth, X. Wang, T. Laoui, and L. Froyen, Lasers and Materials in Selective Laser Sintering, Assem. Autom., 2003, 23(4), p 357-371

20. P. Dudek, FDM 3D Printing Technology in Manufacturing Composite Elements, Arch. Metall. Mater., 2013, 58(4), p 1415-1418

21. M. Prechtl, A. Otto, and M. Geiger, Rapid tooling by laminated object manufacturing of metal foil, Advanced Materials Research, Trans Tech Publ, Switzerland, 2005, p 303-312

22. G. Postiglione, G. Natale, G. Griffini, M. Levi, and S. Turri, Conductive 3D Microstructures by Direct 3D Printing of Polymer/ Carbon Nanotube Nanocomposites Via Liquid Deposition Modeling, Compos. A Appl. Sci. Manuf., 2015, 76, p 110-114

23. K. Gnanasekaran, T. Heijmans, S. van Bennekom, H. Woldhuis, S. Wijnia, G. de With, and H. Friedrich, 3D Printing of CNT- and Graphene-Based Conductive Polymer Nanocomposites by Fused Deposition Modeling, Appl. Mater. Today, 2017, 9, p 21-28

24. S. Dul, L. Fambri, and A. Pegoretti, Fused Deposition Modelling with ABS-Graphene Nanocomposites, Compos. A Appl. Sci. Manuf., 2016, 85, p 181-191

25. A. Dorigato, V. Moretti, S. Dul, S. Unterberger, and A. Pegoretti, Electrically Conductive Nanocomposites for Fused Deposition Modelling, Synth. Met., 2017, 226, p 7-14

26. D. Rigotti, L. Fambri, and A. Pegoretti, Polyvinyl Alcohol Reinforced with Carbon Nanotubes for Fused Deposition Modeling, J. Reinf. Plast. Compos., 2018, 37(10), p 716-727

27. Z. Wu, W. Liu, H. Wu, R. Huang, R. He, Q. Jiang, Y. Chen, X. Ji, Z. Tian, and S. Wu, Research into the Mechanical Properties, Sintering Mechanism and Microstructure Evolution of $\mathrm{Al}_{2} \mathrm{O}_{3}-\mathrm{ZrO}_{2}$ Composites Fabricated by a Stereolithography-Based 3D Printing Method, Mater Chem. Phys., 2018, 207, p 1-10
28. F. Castles, D. Isakov, A. Lui, Q. Lei, C.E.J. Dancer, Y. Wang, J.M. Janurudin, S.C. Speller, C.R.M. Grovenor, and P.S. Grant, Microwave Dielectric Characterisation of 3D-Printed $\mathrm{BaTiO}_{3} / \mathrm{ABS}$ Polymer Composites, Sci. Rep., 2016, 6, p 22714

29. J.J. Martin, B.E. Fiore, and R.M. Erb, Designing Bioinspired Composite Reinforcement Architectures Via 3D Magnetic Printing, Nat. Commun., 2015, 6, p 8641

30. T. Monaghan, A.J. Capel, S.D. Christie, R.A. Harris, and R.J. Friel, Solid-State Additive Manufacturing for Metallized Optical Fiber Integration, Compos. Part A Appl. Sci. Manuf., 2015, 76(3), p 181-193

31. B.G. Compton and J.A. Lewis, 3D-Printing of Lightweight Cellular Composites, Adv. Mater, 2014, 26(34), p 5930-5935

32. S.E. Bakarich, R. Gorkin, III, M. in het Panhuis, and G.M. Spinks, Three-Dimensional Printing Fiber Reinforced Hydrogel Composites, ACS Appl. Mater. Interfaces, 2014, 6(18), p 15998-16006

33. E.D. Yildirim, X. Yin, K. Nair, and W. Sun, Fabrication, Characterization, and Biocompatibility of Single-Walled Carbon NanotubeReinforced Alginate Composite Scaffolds Manufactured Using Freeform Fabrication Technique, J. Biomed. Mater. Res. B Appl. Biomater, 2008, 87(2), p 406-414

34. M. Saari, B. Cox, E. Richer, P.S. Krueger, and A.L. Cohen, Fiber Encapsulation Additive Manufacturing: An Enabling Technology for 3D Printing of Electromechanical Devices and Robotic Components, 3D Print. Addit. Manuf., 2015, 2(1), p 32-39

35. L.S. Dimas, G.H. Bratzel, I. Eylon, and M.J. Buehler, Tough Composites Inspired by Mineralized Natural Materials: Computation, 3D Printing, and Testing, Adv. Funct. Mater., 2013, 23(36), p 46294638

36. L. Li, J. Wang, P. Lin, and H. Liu, Microstructure and Mechanical properties of Functionally Graded TiCp/Ti6Al4V Composite Fabricated by Laser Melting Deposition, Ceram. Int., 2017, 43(18), p $16638-16651$

37. M. Knupfer, Electronic Properties of Carbon Nanostructures, Surf. Sci. Rep., 2001, 42(1), p 1-74

38. A.V. Eletskii, Mechanical Properties of Carbon Nanostructures and Related Materials, Phys. Usp., 2007, 50(3), p 225-261

39. A.A. Balandin, Thermal Properties of Graphene and Nanostructured Carbon Materials, Nat. Mater., 2011, 10(8), p 569-581

40. S.M. O'Flaherty, R. Murphy, S.V. Hold, M. Cadek, J.N. Coleman, and W.J. Blau, Material Investigation and Optical Limiting Properties of Carbon Nanotube and Nanoparticle Dispersions, J. Phys. Chem. B, 2003, 107(4), p 958-964

41. A. Züttel, C. Nützenadel, P. Sudan, P. Mauron, C. Emmenegger, S. Rentsch, L. Schlapbach, A. Weidenkaff, and T. Kiyobayashi, Hydrogen Sorption by Carbon Nanotubes and Other Carbon Nanostructures, J. Alloys Compd., 2002, 330, p 676-682

42. F. Lopez-Urias, J. Rodriguez-Manzo, M. Terrones, and H. Terrones, Magnetic Properties of Carbon Nanostructures, Int. J. Nanotechnol., 2007, 4(6), p 651-666

43. K. Fu, Y. Yao, J. Dai, and L. Hu, Progress in 3D Printing of Carbon Materials for Energy-Related Applications, Adv. Mater, 2017, 29(9), p 1603486

44. J.J. Restrepo and H.A. Colorado, Additive Manufacturing of Epoxy Resin Matrix Reinforced with Magnetic Particles, TMS Annual Meeting \& Exhibition, Springer, Berlin, 2018, p 619-624

45. L. Kuentz, A. Salem, M. Singh, M. Halbig, and J. Salem, Additive Manufacturing and Characterization of Polylactic Acid (PLA) Composites Containing Metal Reinforcements, Proc. 40th International Conference and Expo on Advanced Ceramic and Composites, Datona Beach, Florida, USA, January 24, 2016, I.D. No.20160010284

46. Z. Liu, J. Zhan, M. Fard, and J.L. Davy, Acoustic Properties of a Porous Polycarbonate Material Produced by Additive Manufacturing, Mater. Lett., 2016, 181, p 296-299

47. J. Bai, S. Yuan, F. Shen, B. Zhang, C.K. Chua, K. Zhou, and J. Wei, Toughening of Polyamide 11 with Carbon Nanotubes for Additive Manufacturing, Virt. Phys. Prototyp., 2017, 12(3), p 235-240

48. A. Ambrosi and M. Pumera, 3D-Printing Technologies for Electrochemical Applications, Chem. Soc. Rev., 2016, 45(10), p 2740-2755

49. I. Hanzlicek, M. Pentek, Computational Modeling of Stereolithography, IEEE GSC, 2014

50. Q. Mu, C.K. Dunn, L. Wang, M.L. Dunn, H.J. Qi, and T.J.S.M. Wang, Structures, Thermal Cure Effects on Electromechanical Properties of 
Conductive Wires by Direct Ink Write for 4D Printing and Soft Machines, Smart Mater. Struct., 2017, 26(4), p 045008

51. K.H. Leitz, P. Singer, A. Plankensteiner, B. Tabernig, H. Kestler, and L.S. Sigl, Multi-Physical Simulation of Selective Laser Melting, Met. Powder Rep., 2017, 72(5), p 331-338

52. A. Boschetto and L. Bottini, Accuracy Prediction in Fused Deposition Modeling, Int. J. Adv. Manuf. Technol., 2014, 73(5-8), p 913-928

53. B.N. Turner, R. Strong, and S.A. Gold, A Review of Melt Extrusion Additive Manufacturing Processes: I. Process Design and Modeling, Rapid Prototyp. J., 2014, 20(3), p 192-204

54. X. Wang, M. Jiang, Z. Zhou, J. Gou, and D. Hui, 3D Printing of Polymer Matrix Composites: A Review and Prospective, Compos. B Eng., 2017, 110, p 442-458

55. P. Parandoush and D. Lin, A Review on Additive Manufacturing of Polymer-Fiber Composites, Compos. Struct., 2017, 182, p 36-53

56. U. Scheithauer, A. Bergner, E. Schwarzer, H.-J. Richter, and T. Moritz, Studies on Thermoplastic 3D Printing of Steel-Zirconia Composites, J. Mater. Res., 2014, 29(17), p 1931-1940

57. X. Tian, T. Liu, C. Yang, Q. Wang, and D. Li, Interface and Performance of 3D Printed Continuous Carbon Fiber Reinforced PLA Composites, Compos. A Appl. Sci. Manuf., 2016, 88, p 198-205

58. X. Wei, D. Li, W. Jiang, Z. Gu, X. Wang, Z. Zhang, and Z. Sun, 3D Printable Graphene Composite, Sci. Rep., 2015, 5, p 11181

59. S. Dul, L. Fambri, and A. Pegoretti, Filaments Production and Fused Deposition Modelling of ABS/Carbon Nanotubes Composites, Nanomaterials, 2018, 8(1), p 49

60. K. Prashantha and F. Roger, Multifunctional Properties of 3D Printed Poly(Lactic Acid)/Graphene Nanocomposites by Fused Deposition Modeling, J. Macromol. Sci. Part A, 2017, 54(1), p 24-29

61. S.C. Partain, Fused Deposition Modeling with Localized Pre-deposition Heating Using Forced Air, Montana State University-Bozeman, College of Engineering, Bozeman, 2007

62. C.B. Sweeney, B.A. Lackey, M.J. Pospisil, T.C. Achee, V.K. Hicks, A.G. Moran, B.R. Teipel, M.A. Saed, and M.J. Green, Welding of 3DPrinted Carbon Nanotube-Polymer Composites by Locally Induced Microwave Heating, Sci. Adv., 2017, 3(6), p e1700262

63. F. Ning, W. Cong, Y. Hu, and H. Wang, Additive Manufacturing of Carbon Fiber-Reinforced Plastic Composites Using Fused Deposition Modeling: Effects of Process Parameters on Tensile Properties, J. Compos. Mater, 2017, 51(4), p 451-462

64. H.L. Tekinalp, V. Kunc, G.M. Velez-Garcia, C.E. Duty, L.J. Love, A.K. Naskar, C.A. Blue, and S. Ozcan, Highly Oriented Carbon Fiber-Polymer Composites Via Additive Manufacturing, Compos. Sci. Technol., 2014, 105, p 144-150

65. W. Hao, Y. Liu, H. Zhou, H. Chen, and D. Fang, Preparation and Characterization of 3D Printed Continuous Carbon Fiber Reinforced Thermosetting Composites, Polym. Test., 2018, 65, p 29-34

66. K. Chockalingam, N. Jawahar, K. Ramanathan, and P. Banerjee, Optimization of Stereolithography Process Parameters for Part Strength Using Design of Experiments, Int. J. Adv. Manuf. Technol., 2006, 29(1-2), p 79-88

67. H.K. Park, M. Shin, B. Kim, J.W. Park, and H. Lee, A Visible LightCurable Yet Visible Wavelength-Transparent Resin for Stereolithography 3D Printing, NPG Asia Mater, 2018, 10, p 82-89

68. F.P.W. Melchels, J. Feijen, and D.W. Grijpma, A Review on Stereolithography and Its Applications in Biomedical Engineering, Biomaterials, 2010, 31(24), p 6121-6130

69. U. Kalsoom, A. Peristyy, P. Nesterenko, and B. Paull, A 3D Printable Diamond Polymer Composite: A Novel Material for Fabrication of Low Cost Thermally Conducting Devices, RSC Adv., 2016, 6(44), p 38140-38147

70. A. Chiappone, I. Roppolo, E. Naretto, E. Fantino, F. Calignano, M. Sangermano, and F. Pirri, Study of Graphene Oxide-Based 3D Printable Composites: Effect of the In Situ Reduction, Compos. B Eng., 2017, 124, p 9-15

71. J. Hector Sandoval and R.B. Wicker, Functionalizing Stereolithography Resins: Effects of Dispersed Multi-walled Carbon Nanotubes on Physical Properties, Rapid Prototyp. J., 2006, 12(5), p 292-303

72. J.H. Sandoval, K.F. Soto, L.E. Murr, and R.B. Wicker, Nanotailoring Photocrosslinkable Epoxy Resins with Multi-walled Carbon Nanotubes for Stereolithography Layered Manufacturing, J. Mater. Sci., 2007, 42(1), p 156-165

73. T. Billiet, M. Vandenhaute, J. Schelfhout, S. Van Vlierberghe, and P. Dubruel, A Review of Trends and Limitations in Hydrogel-Rapid
Prototyping for Tissue Engineering, Biomaterials, 2012, 33(26), p 6020-6041

74. H. Korhonen, L.H. Sinh, N.D. Luong, P. Lehtinen, T. Verho, J. Partanen, J. Seppälä, Fabrication of graphene-based 3D structures by stereolithography, physica status solidi (a), 213(4), 982-985 (2016)

75. D. Lin, S. Jin, F. Zhang, C. Wang, Y. Wang, C. Zhou, and G.J. Cheng, 3D Stereolithography Printing of Graphene Oxide Reinforced Complex Architectures, Nanotechnology, 2015, 26(43), p 434003

76. B.Z. Jang, J.H. Liu, S. Chen, Z.M. Li, H. Mahfuz, A. Adnan, Nanotube fiber reinforced composite materials and method of producing fiber reinforced composites, ed., Google Patents, 2005

77. A. Gupta and A. Ogale, Dual Curing of Carbon Fiber Reinforced Photoresins for Rapid Prototyping, Polym. Compos., 2002, 23(6), p 1162-1170

78. S. Kumar and J.P. Kruth, Composites by Rapid Prototyping Technology, Mater. Des., 2010, 31(2), p 850-856

79. R.L. Truby and J.A. Lewis, Printing Soft Matter in Three Dimensions, Nature, 2016, 540(7633), p 371

80. J.A. Lewis, Direct ink Writing of 3D Functional Materials, $A d v$. Funct. Mater., 2006, 16(17), p 2193-2204

81. J.P. Lewicki, J.N. Rodriguez, C. Zhu, M.A. Worsley, A.S. Wu, Y. Kanarska, J.D. Horn, E.B. Duoss, J.M. Ortega, and W. Elmer, 3DPrinting of Meso-Structurally Ordered Carbon Fiber/Polymer Composites with Unprecedented Orthotropic Physical Properties, Sci. Rep., 2017, 7, p 43401

82. J.J. Guo and J.A. Lewis, Aggregation Effects on the Compressive Flow Properties and Drying Behavior of Colloidal Silica Suspensions, J. Am. Ceram. Soc., 1999, 82(9), p 2345-2358

83. L. Jacot-Descombes, M. Gullo, V. Cadarso, and J. Brugger, Fabrication of Epoxy Spherical Microstructures by Controlled Drop-onDemand Inkjet Printing, J. Micromech. Microeng., 2012, 22(7), p 074012

84. D. Kokkinis, M. Schaffner, and A.R. Studart, Multimaterial Magnetically Assisted 3D Printing of Composite Materials, Nat. Communi., 2015,6, p 8643

85. H.-G. Yi, H. Lee, and D.-W. Cho, 3D Printing of Organs-on-Chips, Bioengineering, 2017, 4(1), p 10

86. B. Dorj, J.E. Won, J.H. Kim, S.J. Choi, U.S. Shin, and H.W. Kim, Robocasting Nanocomposite Scaffolds of Poly (Caprolactone)/Hydroxyapatite Incorporating Modified Carbon Nanotubes for Hard Tissue Reconstruction, J. Biomed. Mater. Res. Part A, 2013, 101(6), p 1670-1681

87. B.G. Compton, N.S. Hmeidat, R.C. Pack, M.F. Heres, and J.R. Sangoro, Electrical and Mechanical Properties of 3D-Printed Graphene-Reinforced Epoxy, JOM, 2018, 70(3), p 292-297

88. A. Denneulin, J. Bras, A. Blayo, B. Khelifi, F. Roussel-Dherbey, and C. Neuman, The Influence of Carbon Nanotubes in Inkjet Printing of Conductive Polymer Suspensions, Nanotechnology, 2009, 20(38), p 385701

89. H.-P. Cong, X.-C. Ren, P. Wang, and S.-H. Yu, Flexible GraphenePolyaniline Composite Paper for High-Performance Supercapacitor, Energy Environ. Sci., 2013, 6(4), p 1185-1191

90. W. Zhu, C. Yan, Y. Shi, S. Wen, J. Liu, Q. Wei, and Y. Shi, A Novel Method Based on Selective Laser Sintering for Preparing HighPerformance Carbon Fibres/Polyamide12/Epoxy Ternary Composites, Sci. Rep., 2016, 6, p 33780

91. C. Yan, L. Hao, L. Xu, and Y. Shi, Preparation, Characterisation and Processing of Carbon Fibre/Polyamide-12 Composites for Selective Laser Sintering, Compos. Sci. Technol., 2011, 71(16), p 1834-1841

92. M. Agarwala, D. Bourell, J. Beaman, H. Marcus, and J. Barlow, Direct Selective Laser Sintering of Metals, Rapid Prototyp. J., 1995, 1(1), p 26-36

93. S. Yuan, Y. Zheng, C.K. Chua, Q. Yan, and K. Zhou, Electrical and Thermal Conductivities of MWCNT/Polymer Composites Fabricated by Selective Laser Sintering, Compos. A Appl. Sci. Manuf., 2018, 105, p 203-213

94. S.R. Athreya, K. Kalaitzidou, and S. Das, Processing and Characterization of a Carbon Black-Filled Electrically Conductive Nylon-12 Nanocomposite Produced by Selective Laser Sintering, Mater. Sci. Eng. A, 2010, 527(10), p 2637-2642

95. M. Chapiro, Current Achievements and Future Outlook for Composites in 3D Printing, Reinf. Plast., 2016, 60(6), p 372-375 
96. Y. Hagedorn, Laser additive manufacturing of ceramic components: materials, processes, and mechanisms, Laser Additive Manufacturing, M. Brandt, Ed., Woodhead Publishing, Cambridge, 2017, p 163-180

97. A. Pc, Micro and Nano Fabrication by Powder Metallurgy, J. Powder Metall. Mininged., 2015, 6, p 8

98. B. Chen, S. Berretta, K. Evans, K. Smith, and O. Ghita, A Primary Study into Graphene/Polyether Ether Ketone (PEEK) Nanocomposite for Laser Sintering, Appl. Surf. Sci., 2018, 428, p 1018-1028

99. J. Azadmanjiri, V.K. Srivastava, P. Kumar, J. Wang, and A. Yu, Graphene-Supported 2D Transition Metal Oxide Heterostructures, J. Mater. Chem. A, 2018, 6, p 13509-13537

100. A.K. Geim, Graphene: Status and Prospects, Science, 2009, 324(5934), p 1530-1534

101. K.S. Novoselov, V. Fal, L. Colombo, P. Gellert, M. Schwab, and K Kim, A Roadmap for Graphene, Nature, 2012, 490(7419), p 192

102. L. Feng, L. Wu, and X. Qu, New Horizons for Diagnostics and Therapeutic Applications of Graphene and Graphene Oxide, Adv. Mater, 2013, 25(2), p 168-186

103. Y. Zhu, S. Murali, W. Cai, X. Li, J.W. Suk, J.R. Potts, and R.S. Ruoff, Graphene and Graphene Oxide: Synthesis, Properties, and Applications, Adv. Mater., 2010, 22(35), p 3906-3924

104. K.P. Loh, Q. Bao, G. Eda, and M. Chhowalla, Graphene Oxide as a Chemically Tunable Platform for Optical Applications, Nat. Chem., 2010, 2(12), p 1015

105. F. Bonaccorso, L. Colombo, G. Yu, M. Stoller, V. Tozzini, A.C. Ferrari, R.S. Ruoff, and V. Pellegrini, Graphene, Related TwoDimensional Crystals, and Hybrid Systems for Energy Conversion and Storage, Science, 2015, 347(6217), p 1246501

106. P. Suvarnaphaet and S.J.S. Pechprasarn, Graphene-Based Materials for Biosensors: A Review, Sensors, 2017, 17(10), p 216

107. B.J. Schultz, R.V. Dennis, V. Lee, and S.J.N. Banerjee, An Electronic Structure Perspective of Graphene Interfaces, Nanoscale, 2014, 6(7), p 3444-3466

108. M. Baudisch, A. Marini, J.D. Cox, T. Zhu, F. Silva, S. Teichmann, M Massicotte, F. Koppens, L.S. Levitov, and F.J.G.J.N.C. de Abajo, Ultrafast Nonlinear Optical Response of Dirac Fermions in Graphene, Nat. Commun., 2018, 9(1), p 1018

109. K. Fu, Y. Wang, C. Yan, Y. Yao, Y. Chen, J. Dai, S. Lacey, Y Wang, J. Wan, and T. Li, Graphene Oxide-Based Electrode Inks for 3D-Printed Lithium-Ion Batteries, Adv. Mater., 2016, 28(13), p 2587-2594

110. T.K. Das and S. Prusty, Graphene-Based Polymer Composites And Their Applications, Polym. Plast. Technol. Eng., 2013, 52(4), p 319 331

111. A.E. Jakus, E.B. Secor, A.L. Rutz, S.W. Jordan, M.C. Hersam, and R.N. Shah, Three-Dimensional Printing of High-Content Graphene Scaffolds for Electronic and Biomedical Applications, ACS Nano, 2015, 9(4), p 4636-4648

112. P. Feng, Y. Kong, L. Yu, Y. Li, C. Gao, S. Peng, H. Pan, Z. Zhao, and C. Shuai, Molybdenum Disulfide Nanosheets Embedded with Nanodiamond Particles: Co-dispersion Nanostructures as Reinforcements for Polymer Scaffolds, Appl. Mater. Today, 2019, 17, p 216-226

113. C. Shuai, Y. Li, G. Wang, W. Yang, S. Peng, and P. Feng, Surface Modification of Nanodiamond: Toward the Dispersion of Reinforced Phase in Poly-l-Lactic Acid Scaffolds, Int. J. Biol. Macromol., 2019, 126, p 1116-1124

114. C. Shuai, W. Guo, P. Wu, W. Yang, S. Hu, Y. Xia, and P. Feng, A Graphene Oxide-Ag co-Dispersing Nanosystem: Dual Synergistic Effects on Antibacterial Activities and Mechanical Properties of Polymer Scaffolds, Chem. Eng. J., 2018, 347, p 322-333

115. A. Kausar, I. Rafique, and B. Muhammad, Aerospace Application of Polymer Nanocomposite with Carbon Nanotube, Graphite, Graphene Oxide, and Nanoclay, Polym. Plast. Technol. Eng., 2017, 56(13), p $1438-1456$

116. H.C. Kim, H.T. Hahn, and Y.S. Yang, Synthesis of PA12/Functionalized GNP Nanocomposite Powders for the Selective Laser Sintering Process, J. Compos. Mater, 2013, 47(4), p 501-509

117. M.F. De Volder, S.H. Tawfick, R.H. Baughman, and A.J. Hart, Carbon Nanotubes: Present and Future Commercial Applications, Science, 2013, 339(6119), p 535-539

118. M.S. Dresselhaus, A. Jorio, M. Hofmann, G. Dresselhaus, and R. Saito, Perspectives on Carbon Nanotubes and Graphene Raman Spectroscopy, Nano Lett., 2010, 10(3), p 751-758
119. B. Peng, M. Locascio, P. Zapol, S. Li, S.L. Mielke, G.C. Schatz, and H.D. Espinosa, Measurements of Near-Ultimate Strength for Multiwalled Carbon Nanotubes and Irradiation-Induced Crosslinking Improvements, Nat. Nanotechnol., 2008, 3(10), p 626

120. E. Pop, D. Mann, Q. Wang, K. Goodson, and H. Dai, Thermal Conductance of an Individual Single-Wall Carbon Nanotube Above Room Temperature, Nano Lett., 2006, 6(1), p 96-100

121. D. Janas and G. Stando, Unexpectedly Strong Hydrophilic Character of Free-Standing Thin Films from Carbon Nanotubes, Sci. Rep., 2017, 7(1), p 12274

122. A. Ihsanullah, A.M. Abbas, T. Al-Amer, M.J. Laoui, M.S. Al-Marri, M. Nasser, and M.A. Khraisheh, Atieh, Heavy Metal Removal from Aqueous Solution by Advanced Carbon Nanotubes: Critical Review of Adsorption Applications, Sep. Purif. Technol., 2016, 157, p 141-161

123. M. Burghard, H. Klauk, and K.J.A.M. Kern, Carbon-Based FieldEffect Transistors for Nanoelectronics, Adv. Mater, 2009, 21(25-26), p 2586-2600

124. R.E.E. Shalin, Polymer Matrix Composites, Springer, Berlin, 2012

125. M. Cadek, J. Coleman, V. Barron, K. Hedicke, and W. Blau, Morphological and Mechanical Properties of Carbon-NanotubeReinforced Semicrystalline and Amorphous Polymer Composites, Appl. Phys. Lett., 2002, 81(27), p 5123-5125

126. K.-T. Hsiao, J. Alms, and S.G. Advani, Use of Epoxy/Multiwalled Carbon Nanotubes as Adhesives to Join Graphite Fibre Reinforced Polymer Composites, Nanotechnology, 2003, 14(7), p 791

127. J.N. Coleman, U. Khan, W.J. Blau, and Y.K. Gunko, Small But Strong: a Review of the Mechanical Properties of Carbon NanotubePolymer Composites, Carbon, 2006, 44(9), p 1624-1652

128. Z. Spitalsky, D. Tasis, K. Papagelis, and C. Galiotis, Carbon Nanotube-Polymer Composites: Chemistry, Processing, Mechanical and Electrical Properties, Prog. Polym. Sci., 2010, 35(3), p 357-401

129. W. Bauhofer and J.Z. Kovacs, A Review and Analysis of Electrical Percolation in Carbon Nanotube Polymer Composites, Compos. Sci. Technol., 2009, 69(10), p 1486-1498

130. W. Yu, H. Zhou, B.Q. Li, and S. Ding, 3D Printing of Carbon Nanotubes-Based Microsupercapacitors, ACS Appl. Mater. Interfaces, 2017, 9(5), p 4597-4604

131. X. Li, R. Cui, W. Liu, L. Sun, B. Yu, Y. Fan, Q. Feng, F. Cui, and F. Watari, The Use of Nanoscaled Fibers or Tubes to Improve Biocompatibility and Bioactivity of Biomedical Materials, J. Nanomater., 2013, 2013, p 14

132. M.A. Correa-Duarte, N. Wagner, J. Rojas-Chapana, C. Morsczeck, M. Thie, and M. Giersig, Fabrication and Biocompatibility of Carbon Nanotube-Based 3D Networks as Scaffolds for Cell Seeding and Growth, Nano Lett., 2004, 4(11), p 2233-2236

133. B.S. Harrison and A. Atala, Carbon Nanotube Applications for Tissue Engineering, Biomaterials, 2007, 28(2), p 344-353

134. L.P. Zanello, B. Zhao, H. Hu, and R.C. Haddon, Bone Cell Proliferation on Carbon Nanotubes, Nano Lett., 2006, 6(3), p 562-567

135. A. Abarrategi, M.C. Gutiérrez, C. Moreno-Vicente, M.J. Hortigüela, V. Ramos, J.L. López-Lacomba, M.L. Ferrer, and F. del Monte, Multiwall Carbon Nanotube Scaffolds for Tissue Engineering Purposes, Biomaterials, 2008, 29(1), p 94-102

136. F.M. Tonelli, A.K. Santos, K.N. Gomes, E. Lorencon, S. Guatimosim, L.O. Ladeira, and R.R. Resende, Carbon Nanotube Interaction with Extracellular Matrix Proteins Producing Scaffolds for Tissue Engineering, Int. J. Nanomed., 2012, 7, p 4511

137. W. Wang, Y. Zhu, S. Liao, and J. Li, Carbon Nanotubes Reinforced Composites for Biomedical Applications, BioMed Res. Int., 2014, 2014, p 8

138. P. Feng, S. Peng, P. Wu, C. Gao, W. Huang, Y. Deng, T. Xiao, and C. Shuai, A Nano-Sandwich Construct Built with Graphene Nanosheets and Carbon Nanotubes Enhances Mechanical Properties of Hydroxyapatite-Polyetheretherketone Scaffolds, Int. J. Nanomed., 2016, 11, p 3487

139. G. Jin and G. Kim, The Effect of Sinusoidal AC Electric Stimulation of 3D PCL/CNT and PCL/ $\beta$-TCP Based Bio-Composites on Cellular Activities for Bone Tissue Regeneration, J. Mater. Chem. B, 2013, 1(10), p 1439-1452

140. F.H. Gojny, M.H. Wichmann, B. Fiedler, and K. Schulte, Influence of Different Carbon Nanotubes on the Mechanical Properties of Epoxy Matrix Composites-A Comparative Study, Compos. Sci. Technol., 2005, 65(15-16), p 2300-2313 
141. F. Gojny, M. Wichmann, U. Köpke, B. Fiedler, and K. Schulte, Carbon Nanotube-Reinforced Epoxy-Composites: Enhanced Stiffness and Fracture Toughness at Low Nanotube Content, Compos. Sci. Technol., 2004, 64(15), p 2363-2371

142. M. Zhang, X. Song, W. Grove, E. Hull, Z. Pei, F. Ning, and W. Cong, Carbon nanotube reinforced fused deposition modeling using microwave irradiation, ASME 2016 11th International Manufacturing Science and Engineering Conference, 2016, American Society of Mechanical Engineers, pp. V003T008A007-V003T008A007

143. F. Naya, C. González, C.S. Lopes, S. Van der Veen, and F. Pons, Computational Micromechanics of the Transverse and Shear Behavior of Unidirectional Fiber Reinforced Polymers Including Environmental Effects, Compos. A Appl. Sci. Manuf., 2017, 92, p 146-157

144. K. Schulte, S. Chandrasekaran, C. Viets, and B. Fiedler, New functions in polymer composites using a nanoparticle-modified matrix, Multifunctionality of Polymer Compositesed, Vol 30, K. Friedrich and U. Breuer, Ed., William Andrew Publishing, Burlington, 2015, p 875-902

145. A.T. DiBenedetto and L. Pinatti, Harnessing the properties of fiberreinforced composites in the design of tissue-engineered scaffolds, Biomedical Compositesed, L. Ambrosio, Ed., Woodhead Publishing, Switzerland, 2010, p 296-322

146. J. Wen, Z. Xia, and F. Choy, Damage Detection of Carbon Fiber Reinforced Polymer Composites Via Electrical Resistance Measurement, Compos. B Eng., 2011, 42(1), p 77-86

147. A. Razaq, L. Nyholm, M. Sjödin, M. Strømme, and A. Mihranyan, Paper-Based Energy-Storage Devices Comprising Carbon FiberReinforced Polypyrrole-Cladophora Nanocellulose Composite Electrodes, Adv. Energy Mater., 2012, 2(4), p 445-454

148. S.J. Park and M.K. Seo, Carbon Fiber-Reinforced Polymer Composites: Preparation, Properties, and Applications, Polym. Compos., 2012, 12, p 135-183
149. D.D. Chung and D. Chung, Carbon Fiber Composites, Elsevier, Amsterdam, 2012

150. B.A. Newcomb and H.G. Chae, The properties of carbon fibers, Handbook of Properties of Textile and Technical Fibres, 2nd ed., A.R. Bunsell, Ed., Woodhead Publishing, Switzerland, 2018, p 841-871

151. S.M.J.I. Dambrot, Chemistry Helps Athlete Keep Moving, ChemMaters, 2016, 2015, p 20

152. Z. Xu and C.J.M.T. Gao, Graphene Fiber: A New Trend in Carbon Fibers, Mater. Today, 2015, 18(9), p 480-492

153. F. Derbyshire, R. Andrews, D. Jacques, M. Jagtoyen, G. Kimber, and T.J.F. Rantell, Synthesis of Isotropic Carbon fibers and Activated Carbon Fibers from Pitch Precursors, Fuel, 2001, 80(3), p 345-356

154. S.-Y. Fu and B. Lauke, Effects of Fiber Length and Fiber Orientation Distributions on the Tensile Strength of Short-Fiber-Reinforced Polymers, Compos. Sci. Technol., 1996, 56(10), p 1179-1190

155. F. Van Der Klift, Y. Koga, A. Todoroki, M. Ueda, Y. Hirano, and R. Matsuzaki, 3D Printing of Continuous Carbon Fibre Reinforced Thermo-Plastic (CFRTP) Tensile Test Specimens, Open J. Compos. Mater, 2016, 6(1), p 18-27

156. G.D. Goh, V. Dikshit, A.P. Nagalingam, G.L. Goh, S. Agarwala, S.L. Sing, J. Wei, and W.Y. Yeong, Characterization of Mechanical Properties and Fracture Mode of Additively Manufactured Carbon Fiber and Glass Fiber Reinforced Thermoplastics, Mater. Des., 2018, 137, p 79-89

157. A.N. Dickson, J.N. Barry, K.A. McDonnell, and D.P. Dowling, Fabrication of Continuous Carbon, Glass and Kevlar Fibre Reinforced Polymer Composites Using Additive Manufacturing, Addit. Manuf., 2017, 16, p 146-152

Publisher's Note Springer Nature remains neutral with regard to jurisdictional claims in published maps and institutional affiliations. 